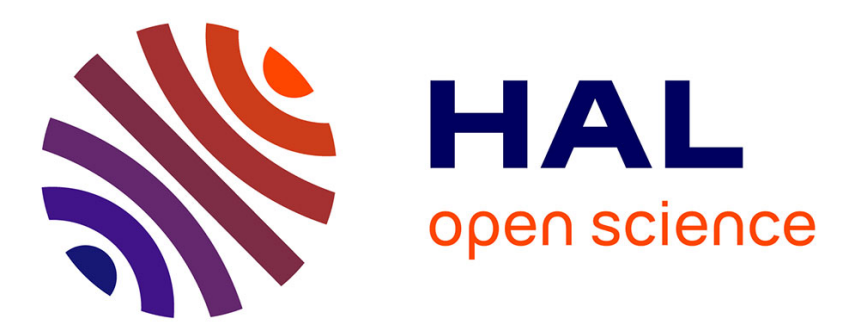

\title{
Estimation of the fuzzy substructure model parameters using the mean power flow equation of the fuzzy structure
}

Christian Soize

\section{- To cite this version:}

Christian Soize. Estimation of the fuzzy substructure model parameters using the mean power flow equation of the fuzzy structure. Journal of Vibration and Acoustics, 1998, 120 (1), pp.279-286. 10.1115/1.2893818 . hal-00765810

\section{HAL Id: hal-00765810 https://hal.science/hal-00765810}

Submitted on 16 Dec 2012

HAL is a multi-disciplinary open access archive for the deposit and dissemination of scientific research documents, whether they are published or not. The documents may come from teaching and research institutions in France or abroad, or from public or private research centers.
L'archive ouverte pluridisciplinaire HAL, est destinée au dépôt et à la diffusion de documents scientifiques de niveau recherche, publiés ou non, émanant des établissements d'enseignement et de recherche français ou étrangers, des laboratoires publics ou privés. 


\title{
Estimation of the Fuzzy Substructure Model Parameters Using the Mean Power Flow Equation of the Fuzzy Structure
}

\author{
C. Soize \\ Structures Department, ONERA, BP 72, F-92322 Chatillon Cedex, France
}

\begin{abstract}
This paper presents a theoretical approach for identifying the dimensionless mean coefficient of participating fuzzy mass which is the main unknown parameter of the type I or II fuzzy law previously introduced by the author. This method is based on the use of the associated power flow equation, each power term being identified by using a global statistical energy analysis of the fuzzy structure (master structure with its fuzzy substructures). Identification is then carried out by solving a nonlinear constrained optimization problem. An example is given to illustrate the theoretical results.
\end{abstract}

\section{Introduction}

In the fuzzy structure theory (Soize, 1986), the concept of "master structure" is introduced to designate the part of the mechanical system which is accessible to conventional modeling. The "fuzzy substructure" is by definition the part of the structure that is not accessible to conventional modeling because details are unknown or are imprecisely known. The fuzzy substructure consists of the secondary mechanical subsystems "attached" to the master structure. A "fuzzy structure" is a master structure plus one or more fuzzy substructures globally called the fuzzy substructure. This theory was developed to explain and model the apparent dissipation occurring on the master structure in order to improve the structural-acoustics predictions of structures with internal structural complexity (Soize, 1986; Chabas et al., 1986; Soize 1993 and 1995). This dissipation is due to the mechanical energy transferred from the master structure to the secondary mechanical subsystems attached to the primary structure and entering in vibration. It is important to note that the objective of the fuzzy structure theory (developed by the author) is to predict the modulus and phase of the response in every point of the master structure, taking the effects of the structural complexity (modeled by a fuzzy substructure described by a probabilistic approach) into account. Accordingly, this theory is not equivalent to Statistical Energy Analysis (SEA) (contrary to what was asserted by Lyon, 1995).

Since 1991, much interesting research has been published in this area by the US acoustical community (see for instance Sparrow, 1991; Russell and Sparrow, 1992; Pierce et al., 1993; Dyer, 1994; McCoy, 1994; Pierce, 1994; Rochat and Sparrow, 1994; Sparrow et al., 1994; Cuschieri and Feit, 1995; Feit and Pierce, 1995; Maidanik, 1995; Maidanik and Dickey, 1995; Pierce, 1995a and 1995b; Photiadis, 1995; Rochat and Sparrow, 1995; Ruckman and Feit 1995a and 1995b; Russell, 1995; Russell and Sparrow, 1995; Sparrow, 1995; Steinberg and McCoy, 1995; Strasberg, 1996; Strasberg and Feit, 1996).

An important problem with this kind of theory is how to determine the parameters to be used in the fuzzy structure laws (see for instance Soize, 1986; Pierce et al. 1993; Feit and Pierce, 1995; Pierce, 1995b; Russell, 1995; Soize, 1995). This paper presents a theoretical approach for identifying the dimensionless mean coefficient of participating fuzzy mass which is the main unknown parameter of the type I or II fuzzy law introduced in 1986 by the author. This method is based on the use of the associated power flow equation, each power term being identified by using a global statistical energy analysis of the fuzzy structure. The identification is then carried 
out by solving a nonlinear constrained optimization problem. An example is given to illustrate the theoretical results.

\section{Modeling of the Master Structure Coupled with its Fuzzy Substructures}

This section deals with modeling of a master structure coupled with its fuzzy substructures to predict vibration. To simplify the presentation, we assume that no external or internal fluid is coupled with the primary structure and consequently that there is an identity between the master structure and the primary structure (for the elastoacoustic case, see Soize, 1993; Soize et al., 1992). The physical space $\mathbb{R}^{3}$ is referenced to a cartesian coordinate system $O x_{1} x_{2} x_{3}$; we denote the generic point of $\mathbb{R}^{3}$ as $\mathbf{x}=\left(x_{1}, x_{2}, x_{3}\right)$. The geometry of the fuzzy structure and the notations are defined in Fig. 1. The master structure is a three-dimensional continuum which occupies the open bounded domain $D_{\text {mast }}$ of $\mathbb{R}^{3}$ with boundary $\partial D_{\text {mast }}=\Sigma_{\text {mast }} \cup \Sigma_{\text {fuz }}$. The fuzzy substructure $D_{\text {fuz }}$ is written as the union $D_{\text {fuz }}=\cup_{\ell=1}^{L} D_{\text {fuz }, \ell}$ of $L \geq 1$ fuzzy substructures. Each fuzzy substructure $D_{\text {fuz, } \ell}$ is attached to the master structure by the boundary $\Gamma_{\ell}$ and there is no mechanical connection between any two fuzzy substructures. The part $\Sigma_{\mathrm{fuz}}=\cup_{\ell=1}^{L} \Gamma_{\ell}$ is the common boundary between the fuzzy substructures and the master structure. The external unit normal to $\partial D_{\text {mast }}$ is denoted $\mathbf{n}$ and the two-dimensional surface area element is denoted $d s$. We then have $\int_{\Gamma_{\ell}} d s(\mathbf{x})=\left|\Gamma_{\ell}\right|$, where $\left|\Gamma_{\ell}\right|$ is the area of surface $\Gamma_{\ell}$.

2.1 Probabilistic Equations of the Fuzzy Structure in the Frequency Domain. The equations are written in the frequency domain $\omega \in \mathbb{R}$. The master structure is modeled by an inhomogeneous, anisotropic, linear viscoelastic solid continuum. Let $\mathbf{u}(\mathbf{x}, \omega)=\left(u_{1}(\mathbf{x}, \omega), u_{2}(\mathbf{x}, \omega)\right.$, $\left.u_{3}(\mathbf{x}, \omega)\right)$ be the $\mathbb{C}^{3}$-valued displacement field of the master structure defined on $D_{\text {mast }}$. Boundary $\Sigma_{\text {mast }}$ is written as $\Gamma_{0} \cup \Gamma_{\text {mast }}$. The master structure is fixed on boundary $\Gamma_{0}$. A given $\mathbb{C}^{3}$ valued surface force field $\mathbf{g}_{\text {surf }}(\mathbf{x}, \omega)=\left(g_{\text {surf }, 1}(\mathbf{x}, \omega), g_{\text {surf }, 2}(\mathbf{x}, \omega), g_{\text {surf }, 3}(\mathbf{x}, \omega)\right)$ is prescribed on boundary $\Gamma_{\text {mast }}$. For $i \in\{1,2,3\}$ the equations of the master structure are

$$
\begin{gathered}
-\omega^{2} \rho u_{i}-\sum_{j} \frac{\partial \sigma_{i j}}{\partial x_{j}}=g_{\mathrm{vol}, i} \text { in } D_{\text {mast }}, \\
u_{i}=0 \quad \text { on } \Gamma_{0}, \\
\sum_{j} \sigma_{i j} n_{j}=g_{\text {surf }, i} \quad \text { on } \Gamma_{\text {mast }}, \\
\sum_{j} \sigma_{i j} n_{j}=-\gamma_{i}^{\ell} \text { on } \Gamma_{\ell}, \text { for } \ell \in\{1, \ldots, L\}
\end{gathered}
$$

in which $\mathbf{g}_{\mathrm{vol}}(\mathbf{x}, \omega)=\left(g_{\mathrm{vol}, 1}(\mathbf{x}, \omega), g_{\mathrm{vol}, 2}(\mathbf{x}, \omega), g_{\mathrm{vol}, 3}(\mathbf{x}, \omega)\right)$ is a given $\mathbb{C}^{3}$-valued volumetric force field applied in $D_{\text {mast }}, \rho(\mathbf{x})$ is the mass density field on $D_{\text {mast }}$ at equilibrium, $\sigma_{i j}$ is the stress tensor and $\gamma^{\ell}(\mathbf{x}, \omega)=\left(\gamma_{1}^{\ell}(\mathbf{x}, \omega), \gamma_{2}^{\ell}(\mathbf{x}, \omega), \gamma_{3}^{\ell}(\mathbf{x}, \omega)\right)$ is the $\mathbb{C}^{3}$-valued surface force field defined on $\Gamma_{\ell}$, representing the action of the master structure on the fuzzy substructure $D_{\mathrm{fuz}, \ell}$ The constitutive law in the frequency domain allows symmetric stress tensor $\sigma_{i j}$ to be related to the linearized symmetric strain tensor $\varepsilon_{i j}(\mathbf{u})=1 / 2\left(\partial u_{i} / \partial x_{j}+\partial u_{j} / \partial x_{i}\right)$ by the equation

$$
\sigma_{i j}(\mathbf{x}, \omega)=\sum_{k, h} a_{i j k h}(\mathbf{x}, \omega) \varepsilon_{k h}(\mathbf{u})+i \omega \sum_{k, h} b_{i j k h}(\mathbf{x}, \omega) \varepsilon_{k h}(\mathbf{u})
$$

in which the real tensor $a_{i j k h}(\mathbf{x}, \omega)$ of the elastic coefficients and the real tensor $b_{i j k h}(\mathbf{x}, \omega)$ of the damping coefficients have the usual properties of symmetry and positive-definiteness. Using 
the fuzzy structure theory (Soize, 1986 and 1993), each surface force field $\gamma^{\ell}(\mathbf{x}, \omega)$ related to the fuzzy substructure attached to $\Gamma_{\ell}$ is modeled by a random field defined by a probabilistic boundary impedance operator such that, for all $\ell \in\{1, \ldots, L\}$,

$$
\gamma^{\ell}(\mathbf{x}, \omega)=\int_{\Gamma_{\ell}} i \omega\left[\mathbf{Z}^{\ell}\left(\mathbf{x}, \mathbf{x}^{\prime}, \omega\right)\right] \mathbf{u}\left(\mathbf{x}^{\prime}, \omega\right) d s\left(\mathbf{x}^{\prime}\right) \text { on } \quad \Gamma_{\ell}
$$

in which $\left[\mathbf{Z}^{\ell}\left(\mathbf{x}, \mathbf{x}^{\prime}, \omega\right)\right]$ is a $(3 \times 3)$ complex random matrix relative to the canonical basis of $\mathbb{R}^{3}$. Let $H$ be the Hilbert space of all the integrable square functions from $D_{\text {mast }}$ into $\mathbb{C}^{3}$, equipped with the inner product $\ll \mathbf{w}, \delta \mathbf{w} \gg=\int_{D_{\text {mast }}}<\mathbf{w}(\mathbf{x}), \overline{\delta \mathbf{w}(\mathbf{x})}>d \mathbf{x}$ where the overline denotes the complex conjugate and $<\mathbf{w}(\mathbf{x}), \delta \mathbf{w}(\mathbf{x})\rangle=\sum_{j} w_{j}(\mathbf{x}) \delta w_{j}(\mathbf{x})$. The random boundary impedance operator $\mathbf{Z}_{\mathrm{fuz}}(\omega)$ of the fuzzy substructure $D_{\mathrm{fuz}}=\cup_{\ell=1}^{L} D_{\mathrm{fuz}, \ell}$ can be written as

$$
\mathbf{Z}_{\text {fuz }}(\omega)=E\left\{\mathbf{Z}_{\text {fuz }}(\omega)\right\}+\mathbf{Z}_{\text {rand }}(\omega),
$$

in which $E\left\{\mathbf{Z}_{\text {fuz }}(\omega)\right\}$ is the mathematical expectation of $\mathbf{Z}_{\text {fuz }}(\omega)$ (mean value) and $\mathbf{Z}_{\text {rand }}(\omega)$ is the centered random fluctuation. The mean boundary impedance operator $E\left\{\mathbf{Z}_{\text {fuz }}(\omega)\right\}$ of the fuzzy substructure is such that

$$
\ll E\left\{\mathbf{Z}_{\mathrm{fuz}}(\omega)\right\} \mathbf{u}(\omega), \delta \mathbf{u} \gg=\sum_{\ell=1}^{L} \int_{\Gamma_{\ell}} \int_{\Gamma_{\ell}}<\left[Z^{\ell}\left(\mathbf{x}, \mathbf{x}^{\prime}, \omega\right)\right] \mathbf{u}\left(\mathbf{x}^{\prime}, \omega\right), \overline{\delta \mathbf{u}(\mathbf{x})}>d s(\mathbf{x}) d s\left(\mathbf{x}^{\prime}\right)
$$

in which the mean value $\left[Z^{\ell}\left(\mathbf{x}, \mathbf{x}^{\prime}, \omega\right)\right]$ of random matrix $\left[\mathbf{Z}^{\ell}\left(\mathbf{x}, \mathbf{x}^{\prime}, \omega\right)\right]$ verifies $\left[Z^{\ell}\left(\mathbf{x}, \mathbf{x}^{\prime}, \omega\right)\right]=$ $\left[Z^{\ell}\left(\mathbf{x}^{\prime}, \mathbf{x}, \omega\right)\right]^{T}$ and $\left[Z^{\ell}\left(\mathbf{x}, \mathbf{x}^{\prime},-\omega\right)\right]=\overline{\left[Z^{\ell}\left(\mathbf{x}^{\prime}, \mathbf{x}, \omega\right)\right]}$, and the right-hand exponent $T$ denotes the transpose ofthe matrix. For any fixed $\omega$ in $\mathbb{R}, E\left\{\mathbf{Z}_{\text {fuz }}(-\omega)\right\}=\overline{E\left\{\mathbf{Z}_{\mathrm{fuz}}(\omega)\right\}}$ and $i \omega E\left\{\mathbf{Z}_{\mathrm{fuz}}(\omega)\right\}$ can be written as

$$
i \omega E\left\{\mathbf{Z}_{\mathrm{fuz}}(\omega)\right\}=-\omega^{2} \mathbf{R}_{\mathrm{fuz}}(\omega)+i \omega \mathbf{I}_{\mathrm{fuz}}(\omega),
$$

in which $\mathbf{R}_{\text {fuz }}(\omega)$ and $\mathbf{I}_{\text {fuz }}(\omega)$ are real linear operators, algebraically symmetric. The fuzzy structure theory (Soize, 1986 and 1993) gives an explicit construction of random fluctuations $\mathbf{Z}_{\text {rand }}(\omega)$ and mean value $E\left\{\mathbf{Z}_{\text {fuz }}(\omega)\right\}$. This construction uses type I and type II fuzzy laws which depend on mechanical parameters. Each mechanical parameter of the fuzzy law is represented by two deterministic values: a mean value which can be viewed as a design value and a value of a dispersion parameter belonging to $[0,1$ [ allowing uncertainties on data (design value) to be introduced. By construction of the fuzzy structure theory, if there are no uncertainties on the mechanical parameters of the fuzzy law (i.e. if the value of each dispersion parameter is zero), then random fluctuation $\mathbf{Z}_{\text {rand }}(\omega)$ is zero. In this paper, we will only use the modeling of $E\left\{\mathbf{Z}_{\text {fuz }}(\omega)\right\}$ and we do not need the modeling of $\mathbf{Z}_{\text {rand }}(\omega)$. In order to facilitate the reading, we summarize the model of $E\left\{\mathbf{Z}_{\mathrm{fuz}}(\omega)\right\}$ in Section 3 .

2.2 Mean Operator Equation of the Master Structure Coupled with its Fuzzy Substructures. We introduce the admissible function space $\mathcal{C}_{\text {ad }}$ of functions $\mathbf{u}$ in $H$, having a generalized derivative in $H$ and satisfying the constraint $\mathbf{u}=\mathbf{0}$ on $\Gamma_{0}$. The variational formulation of the problem defined by Eqs. (1) to (6) yields the random operator equation

$$
i \omega\left(\mathbf{Z}_{\text {mast }}(\omega)+\mathbf{Z}_{\text {fuz }}(\omega)\right) \mathbf{U}(\omega)=\mathbf{f}_{\text {mast }}(\omega),
$$

whose solution $\mathbf{U}(\omega)$ is a random variable with values in $\mathcal{C}_{\text {ad }}$. For all $\delta \mathbf{u}$ in $\mathcal{C}_{\text {ad }}$, element $\mathbf{f}_{\text {mast }}(\omega)$ is defined by

$$
\ll \mathbf{f}_{\text {mast }}(\omega), \delta \mathbf{u} \gg=\int_{D_{\text {mast }}}<\mathbf{g}_{\mathrm{vol}}(\mathbf{x}, \omega), \overline{\delta \mathbf{u}(\mathbf{x})}>d \mathbf{x}+\int_{\Gamma_{\text {mast }}}<\mathbf{g}_{\text {surf }}(\mathbf{x}, \omega), \overline{\delta \mathbf{u}(\mathbf{x})}>d s(\mathbf{x})
$$


Impedance operator $\mathbf{Z}_{\text {mast }}(\omega)$ of the master structure is a linear operator defined on $\mathcal{C}_{\text {ad }}$ by

$$
i \omega \mathbf{Z}_{\text {mast }}(\omega)=-\omega^{2} \mathbf{M}_{\text {mast }}+i \omega \mathbf{C}_{\text {mast }}(\omega)+\mathbf{K}_{\text {mast }}(\omega)
$$

verifying $\mathbf{Z}_{\text {mast }}(-\omega)=\overline{\mathbf{Z}_{\text {mast }}(\omega)}$, and where $\mathbf{M}_{\text {mast }}, \mathbf{C}_{\text {mast }}(\omega)$ and $\mathbf{K}_{\text {mast }}(\omega)$ are real operators, algebraically symmetric and positive-definite, such that, for all $\mathbf{u}$ and $\delta \mathbf{u}$ in $\mathcal{C}_{\mathrm{ad}}$,

$$
\begin{gathered}
\ll \mathbf{M}_{\text {mast }} \mathbf{u}(\omega), \delta \mathbf{u} \gg=\int_{D_{\text {mast }}} \rho(\mathbf{x})<\mathbf{u}(\mathbf{x}, \omega), \overline{\delta \mathbf{u}(\mathbf{x})}>d \mathbf{x}, \\
\ll \mathbf{C}_{\text {mast }}(\omega) \mathbf{u}(\omega), \delta \mathbf{u} \gg=\int_{D_{\text {mast }}} b_{i j k h}(\mathbf{x}, \omega) \varepsilon_{k h}(\mathbf{u}) \varepsilon_{i j}(\overline{\delta \mathbf{u}}) d \mathbf{x} \\
\ll \mathbf{K}_{\text {mast }}(\omega) \mathbf{u}(\omega), \delta \mathbf{u} \gg=\int_{D_{\text {mast }}} a_{i j k h}(\mathbf{x}, \omega) \varepsilon_{k h}(\mathbf{u}) \varepsilon_{i j}(\overline{\delta \mathbf{u}}) d \mathbf{x} .
\end{gathered}
$$

For all fixed real $\omega$, random solution $\mathbf{U}(\omega)$ of Eq. (10) can be written as

$$
\mathbf{U}(\omega)=\mathbf{u}(\omega)+\mathbf{u}_{\text {rand }}(\omega)
$$

in which $\mathbf{u}(\omega)$ is the solution in $\mathcal{C}_{\text {ad }}$ of the following mean operator equation

$$
i \omega\left(\mathbf{Z}_{\text {mast }}(\omega)+E\left\{\mathbf{Z}_{\text {fuz }}(\omega)\right\}\right) \mathbf{u}(\omega)=\mathbf{f}_{\text {mast }}(\omega),
$$

and $\mathbf{u}_{\text {rand }}(\omega)$ is the noncentered random variable with values in $\mathcal{C}_{\text {ad }}$. It should be noted that $E\{\mathbf{U}(\omega)\}=\mathbf{u}(\omega)+E\left\{\mathbf{u}_{\text {rand }}(\omega)\right\}$ and consequently, solution $\mathbf{u}(\omega)$ of mean operator equation (15) does not represent the mean value of the response. The fuzzy structure theory (Soize, 1986 and 1993) gives an explicit construction of random solution $\mathbf{U}(\omega)$ of Eq. (10) (i.e. the construction of $\mathbf{u}(\omega)$ and $\mathbf{u}_{\text {rand }}(\omega)$ using a Neumann expansion of the random linear operator $(\mathbf{I}+\mathbf{T}(\omega))^{-1}$ with $\mathbf{T}(\omega)=\left(\mathbf{Z}_{\text {mast }}(\omega)+E\left\{\mathbf{Z}_{\text {fuz }}(\omega)\right\}\right)^{-1} \mathbf{Z}_{\text {rand }}(\omega)$. Since the present paper is devoted to identification of the dimensionlessmean coefficient of the participating fuzzy mass which is the main unknown mechanical parameter of the type II fuzzy law (Soize, 1995), it is coherent to consider that no uncertainties are present on this mechanical parameter (once the mean value of this mechanical parameter has been identified, uncertainties can be introduced using a nonzero value of the dispersion parameters, and then random Eq. (10) can then be solved. As was recalled in Section 2.1, if there are no uncertainties on the mechanical parameters of the type II fuzzy law, then $\mathbf{Z}_{\text {rand }}(\omega)=0$ and in this case $\mathbf{u}(\omega)=E\{\mathbf{U}(\omega)\}$ represents the mean response of the master structure coupled with its fuzzy substructures (with no uncertainties on the mechanical parameters). Consequently, it is consistent to use the mean power flow equation of the fuzzy structure with no uncertainties on the mechanical parameters of the fuzzy law (i.e. the mean power flow equation associated with Eq. (15) for identifying the mean coefficient of the participating fuzzy mass.

\section{Modeling of the Fuzzy Substructures Using the Type II Fuzzy Law}

In this section, we summarize the model of the mean impedance operator $E\left\{\mathbf{Z}_{\mathrm{fuz}}(\omega)\right\}$ of the fuzzy substructure (defined by Eq. (8)) using the renormalization and notation introduced in Soize, 1995. Each fuzzy substructure $D_{\text {fuz }, \ell}$, attached to the master structure $D_{\text {mast }}$ by the boundary $\Gamma_{\ell}$, is assumed to be a homogeneous orthotropic fuzzy substructure which can be described by the type II fuzzy law (see Soize, 1993). It should be noted that an isotropic fuzzy substructure is a particular case of the orthotropic case, and that the type I fuzzy law (see Soize 
1986), which models a fuzzy substructure with no spatial memory effect, is a particular case of the type II law which allows a spatial memory effect to be modeled. For an orthotropic fuzzy substructure $D_{\text {fuz }, \ell}$, there exists (see Soize 1986) an orthogonal $(3 \times 3)$ real matrix $\left[\Phi^{\ell}(\mathbf{x})\right]^{T}$ for transition from the canonical basis to a local orthonormal basis $\left\{\mathbf{e}^{\ell 1}(\mathbf{x}), \mathbf{e}^{\ell 2}(\mathbf{x}), \mathbf{e}^{\ell 3}(\mathbf{x})\right\}$ related to the boundary $\Gamma_{\ell}$ at point $\mathbf{x} \in \Gamma_{\ell}$ such that $\left[Z^{\ell}\left(\mathbf{x}, \mathbf{x}^{\prime}, \omega\right)\right]=\left[\Phi^{\ell}(\mathbf{x})\right]\left[Z_{0}^{\ell}\left(\mathbf{x}, \mathbf{x}^{\prime}, \omega\right)\right]\left[\Phi^{\ell}\left(\mathbf{x}^{\prime}\right)\right]^{T}$ in which $\left[Z_{0}^{\ell}\left(\mathbf{x}, \mathbf{x}^{\prime}, \omega\right)\right]$ is a $(3 \times 3)$ complex diagonal matrix whose elements are $\left[Z_{0}^{\ell}\left(\mathbf{x}, \mathbf{x}^{\prime}, \omega\right)\right]_{j k}=$ $\delta_{j k} z_{k}^{\ell}\left(\mathbf{x}, \mathbf{x}^{\prime}, \omega\right)$, with $\delta_{j k}=0$ if $j \neq k$ and $\delta_{j j}=1$. The impedances $z_{k}^{\ell}\left(\mathbf{x}, \mathbf{x}^{\prime}, \omega\right)$ are such that

$$
i \omega z_{k}^{\ell}\left(\mathbf{x}, \mathbf{x}^{\prime}, \omega\right)=\nu_{k}^{\ell}\left\{-\omega^{2} r_{k}^{\ell}(\omega)+i \omega h_{k}^{\ell}(\omega)\right\} \delta_{\Gamma_{\ell}}\left(\mathbf{x}^{\prime}-\mathbf{x}\right)
$$

in which $\int_{\Gamma_{\ell}} \varphi\left(\mathbf{x}^{\prime}\right) \delta_{\Gamma_{\ell}}\left(\mathbf{x}^{\prime}-\mathbf{x}\right) d s\left(\mathbf{x}^{\prime}\right)=\varphi(\mathbf{x})$ if $\mathbf{x} \in \Gamma_{\ell}$ and where $\nu_{k}^{\ell}$ will soon be defined. Above the cutoff frequency introduced in the type II fuzzy law, if $2 \omega n_{k}^{\ell}>1 / 2$, then we have

$$
\begin{gathered}
h_{k}^{\ell}(\omega)=\frac{m_{k}^{\ell}}{\left|\Gamma_{\ell}\right|} \frac{1}{n_{k}^{\ell}} \mathcal{J}\left(\xi_{k}^{\ell}, 2 \omega n_{k}^{\ell}, \alpha_{k}^{\ell}\right), \\
r_{k}^{\ell}(\omega)=\frac{m_{k}^{\ell}}{\left|\Gamma_{\ell}\right|} \mathcal{E}\left(\xi_{k}^{\ell}, 2 \omega n_{k}^{\ell}, \alpha_{k}^{\ell}\right),
\end{gathered}
$$

in which, for $0<\xi<1$ and $\tau \in[0.5,+\infty$, real-valued functions $\mathcal{J}$ and $\mathcal{E}$ are such that

$$
\begin{gathered}
\mathcal{J}(\xi, \tau, \alpha)=\xi \tau(1-\alpha)+\alpha \mathcal{J}_{0}(\xi, \tau), \\
\mathcal{E}(\xi, \tau, \alpha)=(\alpha-1)\left(1+\frac{1}{3 \tau^{2}}\right)+\alpha \mathcal{E}_{0}(\xi, \tau) .
\end{gathered}
$$

Real-valued function $\mathcal{J}_{0}$ is defined by

$$
\mathcal{J}_{0}(\xi, \tau)=\frac{\tau^{2}}{8 \sqrt{1-\xi^{2}}}\left\{\arctan Y_{+}(\xi, \tau)-\arctan Y_{-}(\xi, \tau)\right\}
$$

in which $Y_{ \pm}(\xi, \tau)=\xi^{-1}\left(1-\xi^{2}\right)^{-1 / 2}\left\{\xi^{2}+(1 / \tau \pm 2) /(2 \tau)\right\}$. Real-valued function $\mathcal{E}_{0}$ is defined by

$$
\mathcal{E}_{0}(\xi, \tau)=\frac{2}{\tau}-\frac{1}{4 \sqrt{1-\xi^{2}}} \ln \left\{\frac{N(\xi, \tau)}{D(\xi, \tau)}\right\},
$$

in which $N(\xi, \tau)=\left\{U_{+}(\tau)+W_{+}(\xi, \tau)\right\} \times\left\{U_{-}(\tau)-W_{-}(\xi, \tau)\right\}$ and $D(\xi, \tau)=\left\{U_{+}(\tau)-\right.$ $\left.W_{+}(\xi, \tau)\right\} \times\left\{U_{-}(\tau)+W_{-}(\xi, \tau)\right\}$ with $U_{ \pm}(\tau)=1+(1 \pm 1 / \tau)^{2}$ and $W_{ \pm}(\xi, \tau)=2(1-$ $\left.\xi^{2}\right)^{1 / 2}(1 \pm 1 / \tau)$. For each fuzzy substructure $D_{\text {fuz }, \ell}$ attached to $\Gamma_{\ell}$, we denote as $\mathcal{K}_{\ell} \subseteq\{1,2,3\}$ the subset of the directions in which the fuzzy substructure acts (for instance if the active directions are 1 and $3, \mathcal{K}_{\ell}=\{1,3\}$ ). For each fuzzy substructure $D_{\text {fuz }, \ell}$ and each direction $k \in \mathcal{K}_{\ell}$, the frequency-dependent mean fuzzy input parameters $\nu_{k}^{\ell}, n_{k}^{\ell}, \xi_{k}^{\ell}, \alpha_{k}^{\ell}$ which appear in Eqs. (16) to (18) are defined as follows:

(1)- Dimensionless parameter $\nu_{k}^{\ell} \geq 0$ is the mean coefficient of the participating fuzzy mass (see Soize, 1995) of fuzzy substructure $D_{\text {fuz }, \ell}$ for direction $k$, such that

$$
\mu_{k}^{\ell}=\nu_{k}^{\ell} \frac{m_{k}^{\ell}}{\left|\Gamma_{\ell}\right|}
$$


where $m_{k}^{\ell} \geq 0$ is the total mass acting in direction $k$ for $D_{\text {fuz }, \ell}$ and $\mu_{k}^{\ell} \geq 0$ is the mean equivalent mass per unit measure of surface $\Gamma_{\ell}$ of $D_{\mathrm{fuz}, \ell}$ for direction $k$, introduced in the type I or II fuzzy law (see Soize, 1986 and 1993).

(2)- Parameter $n_{k}^{\ell}>0$ is the mean modal density of fuzzy substructure $D_{\text {fuz }, \ell}$ for direction $k$. For details concerning the calculation of this parameter, see Soize, 1995.

(3)- Parameter $\xi_{k}^{\ell}>0$ is the mean rate of internal dissipation of fuzzy substructure $D_{\text {fuz, } \ell}$ for direction $k$. Generally, we have $\xi_{k}^{\ell} \ll 1$, with typical values of the order of 0.001 to 0.01 .

(4)- Finally, $\alpha_{k}^{\ell}$ is the mean equivalent coupling factor (related to the spatial memory effect) of fuzzy substructure $D_{\mathrm{fuz}, \ell}$ for direction $\beta$. This parameter is such that $0 \leq \alpha_{k}^{\ell} \leq 1$ and for $\alpha_{k}^{\ell}=1$, the model corresponds to the type I fuzzy law. In the other cases, it is the type II fuzzy law.

From Eqs. (8) and (16), we deduce that

$$
i \omega E\left\{\mathbf{Z}_{\mathrm{fuz}}(\omega)\right\}=-\omega^{2} \sum_{\ell=1}^{L} \sum_{k \in \mathcal{K}_{\ell}} \nu_{k}^{\ell} \mathbf{S}_{\mathrm{fuz}}^{\ell k}(\omega)+i \omega \sum_{\ell=1}^{L} \sum_{k \in \mathcal{K}_{\ell}} \nu_{k}^{\ell} \mathbf{C}_{\mathrm{fuz}}^{\ell k}(\omega)
$$

in which linear operators $\mathbf{S}_{\text {fuz }}^{\ell k}(\omega)$ and $\mathbf{C}_{\text {fuz }}^{\ell k}(\omega)$ are such that

$$
\begin{aligned}
\ll \mathbf{S}_{\mathrm{fuz}}^{\ell k}(\omega) \mathbf{u}(\omega), \delta \mathbf{u} \gg=r_{k}^{\ell}(\omega) c^{\ell k}(\mathbf{u}, \delta \mathbf{u}) \\
\ll \mathbf{C}_{\mathrm{fuz}}^{\ell k}(\omega) \mathbf{u}(\omega), \delta \mathbf{u} \gg=h_{k}^{\ell}(\omega) c^{\ell k}(\mathbf{u}, \delta \mathbf{u}) \\
c^{\ell k}(\mathbf{u}, \delta \mathbf{u})=\int_{\Gamma_{\ell}}<\mathbf{u}(\mathbf{x}, \omega), \mathbf{e}^{\ell k}(\mathbf{x})><\overline{\delta \mathbf{u}(\mathbf{x})}, \mathbf{e}^{\ell k}(\mathbf{x})>d s(\mathbf{x})
\end{aligned}
$$

\section{Solution of the Mean Operatorial Equation}

Let $\boldsymbol{\nu}$ be the vector in $\mathbb{R}^{N}, L \leq N \leq 3 L$, with components $\nu_{k}^{\ell}, \ell \in\{1, \ldots, L\}, k \in \mathcal{K}_{\ell}$. From Eqs. (12) and (20), we deduce that Eq. (15) can be rewritten as

$$
\begin{gathered}
\mathbf{A}(\omega ; \boldsymbol{\nu}) \mathbf{u}(\omega)=\mathbf{f}_{\text {mast }}(\omega) \\
\mathbf{A}(\omega ; \boldsymbol{\nu})=-\omega^{2}\left(\mathbf{M}_{\text {mast }}+\sum_{\ell=1}^{L} \sum_{k \in \mathcal{K}_{\ell}} \nu_{k}^{\ell} \mathbf{S}_{\text {fuz }}^{\ell k}(\omega)\right) \\
+i \omega\left(\mathbf{C}_{\text {mast }}(\omega)+\sum_{\ell=1}^{L} \sum_{k \in \mathcal{K}_{\ell}} \nu_{k}^{\ell} \mathbf{C}_{\text {fuz }}^{\ell k}(\omega)\right)+\mathbf{K}_{\text {mast }}(\omega)
\end{gathered}
$$

Taking into account the assumptions, it can be proved that, for all fixed real $\omega$, Eq. (24) has a unique solution $\mathbf{u}(\omega)=\mathbf{T}(\omega ; \boldsymbol{\nu}) \mathbf{f}_{\text {mast }}(\omega)$ in $\mathcal{C}_{\text {ad }}$ with $\mathbf{T}(\omega ; \boldsymbol{\nu})=\mathbf{A}(\omega ; \boldsymbol{\nu})^{-1}$. 


\section{Mean Power Flow Equation of the Fuzzy Structure}

Using Eq. (25), Eq. (24) can be rewitten as

$$
\begin{gathered}
\mathbf{f}_{\text {cons }}(\omega)+\mathbf{f}_{\text {mast,diss }}(\omega)+\sum_{\ell=1}^{L} \mathbf{f}_{\text {fuz,diss }}^{\ell}(\omega)=\mathbf{f}_{\text {mast }}(\omega) \\
\mathbf{f}_{\text {cons }}(\omega)=\left\{-\omega^{2}\left(\mathbf{M}_{\text {mast }}+\sum_{\ell=1}^{L} \sum_{k \in \mathcal{K}_{\ell}} \nu_{k}^{\ell} \mathbf{S}_{\text {fuz }}^{\ell k}(\omega)\right)+\mathbf{K}_{\text {mast }}(\omega)\right\} \mathbf{u}(\omega) \\
\mathbf{f}_{\text {mast,diss }}(\omega)=\left\{i \omega \mathbf{C}_{\text {mast }}(\omega)\right\} \mathbf{u}(\omega) \\
\mathbf{f}_{\text {fuz,diss }}^{\ell}(\omega)=\left\{i \omega \sum_{k \in \mathcal{K}_{\ell}} \nu_{k}^{\ell} \mathbf{C}_{\text {fuz }}^{\ell k}(\omega)\right\} \mathbf{u}(\omega)
\end{gathered}
$$

We assume that $\mathbf{g}_{\text {surf }}(\mathbf{x}, t)$ and $\mathbf{g}_{\mathrm{vol}}(\mathbf{x}, t)$ are $\mathbb{R}^{3}$-valued stochastic fields such that, for any functions $\mathbf{a}(\mathbf{x})$ and $\mathbf{b}(\mathbf{x})$ belonging to $\mathcal{C}_{\mathrm{ad}}, f_{\mathbf{a}}(t)=\ll \mathbf{f}_{\text {mast }}(t)$, $\mathbf{a} \gg$ and $f_{\mathbf{b}}(t)=\ll \mathbf{f}_{\text {mast }}(t), \mathbf{b} \gg$ are limited band stationary second-order stochastic processes indexed by $\mathbb{R}$ with values in $\mathbb{C}$ and centered. In addition, we assume that there exists a cross spectral density function $S_{\mathrm{ab}}(\omega)$, related to the cross correlation function by $R_{\mathbf{a b}}(\theta)=E\left\{f_{\mathbf{a}}(t+\theta) \overline{f_{\mathbf{b}}(t)}\right\}=\int_{\mathbb{R}} e^{i \omega \theta} S_{\mathbf{a b}}(\omega) d \omega$, such that

$$
S_{\mathbf{a b}}(\omega)=f_{0}^{2} \mathbf{1}_{B_{m} \cup \bar{B}_{m}}(\omega) \int_{D_{\text {mast }}} \chi(\mathbf{x})<\mathbf{b}(\mathbf{x}), \overline{\mathbf{a}(\mathbf{x})}>d \mathbf{x},
$$

in which $f_{0}$ is a real number, $\mathbf{1}_{B_{m} \cup \bar{B}_{m}}(\omega)$ is the indicatrix function of limited band $B_{m} \cup \bar{B}_{m}$ with $B_{m}=\left[\omega_{m}, \omega_{m}+\Delta \omega_{m}\right]$ and $\bar{B}_{m}=\left[-\omega_{m}-\Delta \omega_{m},-\omega_{m}\right]$ and $\chi(\mathbf{x})$ is a given bounded positivevalued function on $D_{\text {mast }}$. This model means that stochastic field $\mathbf{f}_{\text {mast }}(\mathbf{x}, t)$ is stationary in time (with limited band $B_{m} \cup \bar{B}_{m}$ ), nonhomogeneous and delta correlated in space with intensity $\chi(\mathbf{x})$. Consequently, $\mathbf{u}(\mathbf{x}, t)$ is a time-stationary stochastic field and from Eq. (26), we deduce the following equality of time-stationary stochastic fields

$$
\mathbf{f}_{\text {cons }}(t)+\mathbf{f}_{\text {mast,diss }}(t)+\sum_{\ell=1}^{L} \mathbf{f}_{\text {fuz,diss }}^{\ell}(t)=\mathbf{f}_{\text {mast }}(t)
$$

For all fixed $t$, we introduce the real-valued random variables

$$
\begin{aligned}
\Pi_{\text {cons }}(t ; \boldsymbol{\nu}) & =\ll \mathbf{f}_{\text {cons }}(t), \partial_{t} \mathbf{u} \gg, \\
\Pi_{\text {mast,diss }}(t ; \boldsymbol{\nu}) & =\ll \mathbf{f}_{\text {mast,diss }}(t), \partial_{t} \mathbf{u} \gg, \\
\Pi_{\text {mast-fuz }}^{\ell}(t ; \boldsymbol{\nu}) & =\ll \mathbf{f}_{\text {fuz,diss }}^{\ell}(t), \partial_{t} \mathbf{u} \gg, \\
\Pi_{\text {mast,in }}(t ; \boldsymbol{\nu}) & =\ll \mathbf{f}_{\text {mast }}(t), \partial_{t} \mathbf{u} \gg,
\end{aligned}
$$

which represent instantaneous powers. For all fixed $t$ in $\mathbb{R}$, we denote the mathematical expectation of random variable $\Pi(t ; \boldsymbol{\nu})$ as $\langle\Pi(\boldsymbol{\nu})\rangle=E\{\Pi(t ; \boldsymbol{\nu})\}$. It can then be proved that $<\Pi_{\text {cons }}(\boldsymbol{\nu})>=0$ and from Eq. (31), we deduce that the mean power flow equation can be written as

$$
<\Pi_{\text {mast,diss }}(\boldsymbol{\nu})>+\sum_{\ell=1}^{L}<\Pi_{\text {mast-fuz }}^{\ell}(\boldsymbol{\nu})>=<\Pi_{\text {mast,in }}(\boldsymbol{\nu})>
$$


in which $<\Pi_{\text {mast,diss }}(\boldsymbol{\nu})>$ is the mean power dissipated in the master structure, $<\Pi_{\text {mast-fuz }}^{\ell}(\boldsymbol{\nu})>$ is the mean power flow from the master structure to fuzzy substructure $D_{\text {fuz }, \ell}$ and $\left\langle\Pi_{\text {mast,in }}(\boldsymbol{\nu})\right\rangle$ is the mean input power of the master structure. In order to calculate the mean powers appearing in Eq. (32), we use the Ritz-Galerkin method which allows approximations to be constructed in a subspace $\mathcal{C}_{n} \subset \mathcal{C}_{\text {ad }}$ of dimension $n \geq 1$. Let $\left\{\mathbf{b}_{\alpha}(\mathbf{x}), \alpha=1, \ldots, n\right\}$ be a basis of $\mathcal{C}_{n}$. Any $\mathbf{u}(t) \in \mathcal{C}_{n} \subset \mathcal{C}_{\text {ad }}$ can be written as $\mathbf{u}(\mathbf{x}, t)=\sum_{\alpha=1}^{n} U_{\alpha}(t) \mathbf{b}_{\alpha}(\mathbf{x})$. It can then be proved that

$$
\begin{gathered}
<\Pi_{\text {mast,diss }}(\boldsymbol{\nu})>=2 f_{0}^{2} \int_{B_{m}} \omega^{2} \operatorname{tr}\left\{[\mathcal{T}(\omega ; \boldsymbol{\nu})]^{*}\left[\mathcal{C}_{\text {mast }}(\omega)\right][\mathcal{T}(\omega ; \boldsymbol{\nu})]\left[S_{\mathcal{F}}(\omega)\right]\right\} d \omega \\
<\Pi_{\text {mast-fuz }}^{\ell}(\boldsymbol{\nu})>=2 f_{0}^{2} \sum_{k=1}^{3} \int_{B_{m}} \nu_{k}^{\ell} \omega^{2} \operatorname{tr}\left\{[\mathcal{T}(\omega ; \boldsymbol{\nu})]^{*}\left[\mathcal{C}_{\text {fuz }}^{\ell k}(\omega)\right][\mathcal{T}(\omega ; \boldsymbol{\nu})]\left[S_{\mathcal{F}}(\omega)\right]\right\} d \omega \\
<\Pi_{\text {mast,in }}(\boldsymbol{\nu})>=2 f_{0}^{2} \Re e \int_{B_{m}} i \omega \operatorname{tr}\left\{[\mathcal{T}(\omega ; \boldsymbol{\nu})]\left[S_{\mathcal{F}}(\omega)\right]\right\} d \omega
\end{gathered}
$$

in which $\operatorname{tr}\{[M]\}=\sum_{j}[M]_{j j}$ denotes the trace of matrix $[M],[M]^{*}=\overline{[M]}^{T}$ denotes the adjoint of matrix $[M], \Re e z$ denotes the real part of complex number $z,[\mathcal{T}(\omega ; \boldsymbol{\nu})]$ is an $(n \times n)$ complex symmetric matrix defined by

$$
[\mathcal{T}(\omega ; \boldsymbol{\nu})]=[\mathcal{A}(\omega ; \boldsymbol{\nu})]^{-1}
$$

where the components of the $(n \times n)$ complex symmetric matrix $[\mathcal{A}(\omega ; \boldsymbol{\nu})]$ are such that

$$
[\mathcal{A}(\omega ; \boldsymbol{\nu})]_{\beta \alpha}=\ll \mathbf{A}(\omega ; \boldsymbol{\nu}) \mathbf{b}_{\alpha}, \mathbf{b}_{\beta} \gg,
$$

with $\mathbf{A}(\omega ; \boldsymbol{\nu})$ given by Eq. $(25),\left[\mathcal{C}_{\text {mast }}(\omega)\right]$ and $\left[\mathcal{C}_{\text {fuz }}^{\ell k}(\omega)\right]$ are $(n \times n)$ real positive symmetric matrices (the first one being positive-definite) defined by

$$
\begin{gathered}
{\left[\mathcal{C}_{\text {mast }}(\omega)\right]_{\beta \alpha}=\ll \mathbf{C}_{\text {mast }}(\omega) \mathbf{b}_{\alpha}, \mathbf{b}_{\beta} \gg,} \\
{\left[\mathcal{C}_{\text {fuz }}^{\ell k}(\omega)\right]_{\beta \alpha}=\ll \mathbf{C}_{\text {fuz }}^{\ell k}(\omega) \mathbf{b}_{\alpha}, \mathbf{b}_{\beta} \gg,}
\end{gathered}
$$

and finally, $\left[S_{\mathcal{F}}(\omega)\right]$ is a $(n \times n)$ complex Hermitian matrix such that $\left[S_{\mathcal{F}}(\omega)\right]_{\beta \alpha}=S_{\mathbf{b}_{\beta} \mathbf{b}_{\alpha}}(\omega)$, i.e. taking Eq. (30) into account,

$$
\left[S_{\mathcal{F}}(\omega)\right]_{\beta \alpha}=f_{0}^{2} \mathbf{1}_{B_{m} \cup \bar{B}_{m}}(\omega) \int_{D_{\text {mast }}} \chi(\mathbf{x})<\mathbf{b}_{\alpha}(\mathbf{x}), \overline{\mathbf{b}_{\beta}(\mathbf{x})}>d \mathbf{x}
$$

which represents the matrix-valued spectral density function of second-order stationary stochastic process $\mathcal{F}(t)=\left(\mathcal{F}_{1}(t), \ldots, \mathcal{F}_{n}(t)\right)$ with $\mathcal{F}_{\alpha}(t)=\ll \mathbf{f}_{\text {mast }}(t), \mathbf{b}_{\alpha} \gg$.

\section{Estimation of the Mean Powers Using Statistical Energy Analysis}

We assume that the mean powers appearing in Eq. (32) can be estimated with an SEA model (Lyon and DeJong, 1995). This means that the master structure coupled with all the fuzzy substructures $D_{\mathrm{fuz}, \ell}$, each of which can be considered as an appropriate union of subsystems, can be modeled by SEA. Consequently, for a given frequency band $B_{m} \cup \bar{B}_{m}$, Statistical Energy Analysis is used to estimate the mean power $<\Pi_{\text {mast,diss }}^{\text {SEA }}>$ dissipated in the master structure, the mean input power $<\Pi_{\text {mast,in }}^{\text {SEA }}>$ related to the master structure and for each fuzzy substructure $D_{\text {fuz }, \ell}$, the mean power flow $<\prod_{\text {mast-fuz }}^{\mathrm{SEA}, \ell}>$ from the master structure to fuzzy substructure $D_{\mathrm{fuz}, \ell}$. By construction, these mean powers verify the SEA mean power flow equation

$$
<\Pi_{\text {mast,diss }}^{\mathrm{SEA}}>+\sum_{\ell=1}^{L}<\Pi_{\text {mast-fuz }}^{\mathrm{SEA}, \ell}>=<\Pi_{\text {mast,in }}^{\mathrm{SEA}}>\quad .
$$




\section{Estimation of the Mean Coefficients of the Participating Fuzzy Mass}

7.1 Definition of a nonlinear constrained optimization problem. An estimate of the mean coefficients $\nu_{k}^{\ell}$ of the participating fuzzy mass can be obtained by solving a global nonlinear constrained optimization problem based on the use of the mean power flow equation. To do so, we introduce the cost function $J(\boldsymbol{\nu})$ from $\mathbb{R}^{N}$ into $\mathbb{R}^{+}$

$$
J(\boldsymbol{\nu})=\sum_{\ell=1}^{L}\left(<\Pi_{\text {mast-fuz }}^{\ell}(\boldsymbol{\nu})>-<\Pi_{\text {mast-fuz }}^{\mathrm{SEA}, \ell}>\right)^{2}
$$

and the space $\mathcal{D}_{N} \subset \mathbb{R}^{N}$ of constraints such that, for all $\boldsymbol{\nu}$ in $\mathcal{D}_{N}$,

$$
\begin{gathered}
\nu_{k}^{\ell} \geq 0 \quad, \quad \ell \in\{1, \ldots, L\} \quad, \quad k \in \mathcal{K}_{\ell}, \\
<\Pi_{\text {mast,diss }}(\boldsymbol{\nu})>-<\Pi_{\text {mast,diss }}^{\mathrm{SEA}}>=0 \\
<\Pi_{\text {mast,in }}(\boldsymbol{\nu})>-<\Pi_{\text {mast,in }}^{\mathrm{SEA}}>=0
\end{gathered}
$$

By construction, the value of $\boldsymbol{\nu}$ is then obtained by minimizing $J(\boldsymbol{\nu})$ on $\mathcal{D}_{N}$, i.e. by solving the following global nonlinear constrained optimization problem

$$
\min _{\boldsymbol{\nu} \in \mathcal{D}_{N}} J(\boldsymbol{\nu})
$$

7.2 Remarks concerning the solution of the constrained optimization problem. First, using the implicit function theorem, it can be proved that, if for each $\ell \in\{1, \ldots, L\}$, the mean power flow $<\Pi_{\text {mast-fuz }}^{\mathrm{SEA},}>$ is sufficiently small (that is to say $<\Pi_{\text {mast-fuz }}^{\mathrm{SEA}, \ell} \rightarrow 0$ ), then the constrained optimization problem defined by Eq. (46) has a unique solution. Secondly, subtracting Eq. (41) from Eq. (32), and using Eqs. (44) and (45), we deduce that, for all $\boldsymbol{\nu}$ in $\mathcal{D}_{N}$, we have

$$
\begin{gathered}
<\Pi_{\text {mast,diss }}(\boldsymbol{\nu})>=<\Pi_{\text {mast,diss }}^{\mathrm{SEA}}>, \\
<\Pi_{\text {mast,in }}(\boldsymbol{\nu})>=<\Pi_{\text {mast,in }}^{\mathrm{SEA}}>, \\
\sum_{\ell=1}^{L}<\Pi_{\text {mast-fuz }}^{\ell}(\boldsymbol{\nu})>=\sum_{\ell=1}^{L}<\Pi_{\text {mast-fuz }}^{\mathrm{SEA}, \ell}>.
\end{gathered}
$$

If $\boldsymbol{\nu}_{\mathrm{opt}} \in \mathcal{D}_{N}$ denotes the solution of Eq. (46), minimization of cost function $J(\boldsymbol{\nu})$ on $\mathcal{D}_{N}$ implies the equivalences

$$
<\Pi_{\text {mast-fuz }}^{\ell}\left(\boldsymbol{\nu}_{\text {opt }}\right)>\simeq<\Pi_{\text {mast-fuz }}^{\mathrm{SEA}, \ell}>\quad, \quad \forall \ell \in\{1, \ldots, L\}
$$

in place of Eq. (49). The equalities $<\Pi_{\text {mast-fuz }}^{\ell}\left(\boldsymbol{\nu}_{\mathrm{opt}}\right)>=<\Pi_{\text {mast-fuz }}^{\mathrm{SEA}, \ell}>$ are verified only if $J\left(\boldsymbol{\nu}_{\text {opt }}\right)=0$, but generally, $J\left(\boldsymbol{\nu}_{\text {opt }}\right)>0$. 
7.3 Solving procedure. Let $\boldsymbol{\nu} \mapsto \mathbf{G}(\boldsymbol{\nu})=\left(G_{1}(\boldsymbol{\nu}), \ldots, G_{N+2}(\boldsymbol{\nu})\right)$ be the function from $\mathbb{R}^{N}$ into $\mathbb{R}^{N+2}$ defined by

$$
\mathbf{G}(\boldsymbol{\nu})=\left[\begin{array}{c}
-\boldsymbol{\nu} \\
\left(<\Pi_{\text {mast,diss }}(\boldsymbol{\nu})>-<\Pi_{\text {mast,diss }}^{\mathrm{SEA}}>\right)^{2} \\
\left(<\Pi_{\text {mast,in }}(\boldsymbol{\nu})>-<\Pi_{\mathrm{mast}, \text { in }}^{\mathrm{EEA}}>\right)^{2}
\end{array}\right] .
$$

The constrained optimization problem defined by Eq. (46) can then be rewritten as follows

$$
\min _{\mathbf{G}(\boldsymbol{\nu}) \leq 0} J(\boldsymbol{\nu})
$$

in which $\mathbf{G}(\boldsymbol{\nu}) \leq 0$ means that $G_{j}(\boldsymbol{\nu}) \leq 0$ for $j \in\{1, \ldots, N+2\}$. The nonlinear constrained optimization problem defined by Eq. (52) can be solved using the Sequential Quadratic Programming (SQP) method (implemented, for instance, in Matlab's Optimization Toolbox (see Grace, 1992)).

\section{Validation with an Example}

8.1 Master structure. It is a simply supported beam with a constant cross-sectional area, length $20 \mathrm{~m}$, total mass $20000 \mathrm{~kg}$, Young's modulus $2.1 \times 10^{11} \mathrm{~N} / \mathrm{m}^{2}$, bending inertia $0.001 \mathrm{~m}^{4}$ and a constant structural damping rate 0.01 . The frequency band of analysis is $B=[0,200] \mathrm{Hz}$. The beam is excited in bending mode in the $\left(x_{1}, x_{3}\right)$ plane by a force applied at $x_{1}=7$, with a unit flat spectrum over all the band $B$ of bandwidth $\Delta \omega=2 \pi \times 200 \mathrm{rad} / \mathrm{s}$. Function $\chi(\mathbf{x})$ appearing in Eq. (30) is the mass density of the master structure and $f_{0}=1$.

8.2 Fuzzy substructures. There are two fuzzy substructures attached to the beam on $\Gamma_{1}=$ $\left\{x_{1} ; 4 \leq x_{1} \leq 8\right\}$ and $\Gamma_{2}=\left\{x_{1} ; 12 \leq x_{1} \leq 18\right\}$. Each fuzzy substructure consists of a large number of simple linear oscillators acting in direction $x_{3}$. All the oscillators have the same mass $m_{0}=1.8 \mathrm{~kg}$ and the same damping date $\xi=0.002$. There are $N_{1}=484$ oscillators on $\Gamma_{1}$ and $N_{2}=729$ on $\Gamma_{2}$. The eigenfrequency of each oscillator and the location of its attachment point on the beam are uniformly distributed. The total mass of the fuzzy substructure is 2183 $\mathrm{kg}$ compared with $20000 \mathrm{~kg}$ for the master structure.

8.3 Construction of the reference by numerical simulation. The response of the fuzzy structure, i.e. the response of the master structure coupled with its fuzzy substructures (beam coupled with the two sets of oscillators), is calculated by numerical simulation in which the eigenmodes of the master structure are used as the basis $\left\{\mathbf{b}_{\alpha}(\mathbf{x}), \alpha=1, \ldots, n\right\}$ of $\mathcal{C}_{n}$, with $n=12$. This response is called the reference below. The irregular solid lines of Figs. 2 and 3 show the modulus of the transverse acceleration of the beam in points $x_{1}=7$ (Fig. 2) and $x_{1}=15$ (Fig. 3). In order to see the role played by the fuzzy substructures, we have also represented the response of the master structure alone (beam with no oscillators) in Figs. 2 and 3 , with dashed lines.

8.4 Estimation of the mean powers using Statistical Energy Analysis. The frequency band of analysis is written as $B=\cup_{m=1}^{10} B_{m}$ with, for all $m, \Delta \omega_{m}=\Delta \omega / 10$. A classical SEA model was developed for the fuzzy structure (beam coupled with the oscillators). Figure 4 shows $<\Pi_{\text {mast,diss }}^{\text {SEA }}>$ (solid line) and $<\Pi_{\text {mast,in }}^{\text {SEA }}>$ (dashed line). Figure 5 shows the power flow $<\Pi_{\text {mast-fuz }}^{\mathrm{SEA}, 1}>$ (dashed line) and $<\Pi_{\text {mast-fuz }}^{\mathrm{SEA},}>$ (solid line). 
8.5 Estimation of the mean coefficients of the participating fuzzy mass. Each fuzzy substructure can be simulated by a homogeneous, orthotropic fuzzy substructure acting only in direction $x_{3}$. Consequently, we have $L=N=2$ and $\mathcal{K}_{1}=\mathcal{K}_{2}=\{3\}$. There is no spatial memory effect, and the mean equivalent coupling factors are therefore such that $\alpha_{3}^{1}=\alpha_{3}^{2}=1$ (Type I fuzzy law for each fuzzy substructure). The mean modal density, the mean rate of internal dissipation and the total mass of each fuzzy substructure can be estimated directly using the formulas given by Soize, 1995, yielding $n_{3}^{1}=0.0175(\mathrm{rad} / \mathrm{s})^{-1}, n_{3}^{2}=0.0215(\mathrm{rad} / \mathrm{s})^{-1}$, $\xi_{3}^{1}=\xi_{3}^{2}=0.002$ and $m_{3}^{1}=871, m_{3}^{2}=1312$. The mean coefficients $\nu_{3}^{1}$ and $\nu_{3}^{2}$ of the participating fuzzy mass of the fuzzy substructures are estimated by solving the nonlinear constrained optimization problem defined by Eq. (52), using the Sequential Quadratic Programming (SQP) method implemented in Matlab's Optimization Toolbox of Matlab (see Grace, 1992)). The mean powers $\left\langle\Pi_{\text {mast,diss }}(\boldsymbol{\nu})\right\rangle,\left\langle\Pi_{\text {mast-fuz }}^{1}(\boldsymbol{\nu})\right\rangle,\left\langle\Pi_{\text {mast-fuz }}^{2}(\boldsymbol{\nu})\right\rangle$ and $\left\langle\Pi_{\text {mast,in }}(\boldsymbol{\nu})\right\rangle$ are calculated using Eqs. (33) to (35). Figure 6 shows the results $\left(\nu_{3}^{1}\right)_{\mathrm{opt}, m}$ (dashed line) and $\left(\nu_{3}^{2}\right)_{\mathrm{opt}, m}$ (solid line) for $m=1, \ldots, 10$, in which $\left(\nu_{3}^{\ell}\right)_{\mathrm{opt}, m}$ denotes the solution of the nonlinear constrained optimization problem for band $B_{m}$.

8.6 Comparisons between the reference and the response obtained by the fuzzy structure theory. Figures 7 and 8 show the response of the master structure coupled with its fuzzy substructures and give the comparisons between the reference (see Section 8.3) and the response calculated using the fuzzy structure theory presented in Sections 2 to 4 . The mean coefficients of the participating fuzzy mass are calculated in Section 8.5. For this calculation, we used the values $\nu_{3}^{1}=\frac{1}{10} \sum_{m=1}^{10}\left(\nu_{3}^{1}\right)_{\mathrm{opt}, m}=0.0427$ and $\nu_{3}^{2}=\frac{1}{10} \sum_{m=1}^{10}\left(\nu_{3}^{2}\right)_{\mathrm{opt}, m}=0.0386$ in Eqs. (24) and (25). The results shown in Figs. 7 and 8 concern the modulus (in $\mathrm{dB}$ ), the real part and the imaginary part (in linear scale) of the transverse acceleration of the beam at point $x_{1}=7$ (Figs. 7-a, 7-b, 7-c) and at $x_{1}=15$ (Figs. 8-a, 8-b, 8-c). It can be seen that the results given by the fuzzy structure theory (smoothed solid line) and the reference (irregular solid line) are in excellent agreement in terms of modulus and phase.

\section{Conclusion}

We have presented a constructive method for estimating the mean coefficients of the participating fuzzy mass for each fuzzy substructure modeled with a type I or type II fuzzy law. The method is based on the use of a mean power flow equation which is established for a general fuzzy structure modeled by the fuzzy structure theory (developed earlier by the author). The proposed theoretical approach is applied to an example. The results obtained are good. Finally, it should be noted that the theory presented can be viewed as a new method for the coupling between a subdomain modeled by the local elastodynamic equation (for which the local modulus and phases are preserved) with another subdomain modeled by the global mean power equation of the Statistical Energy Analysis (for which the local modulus and phases are lost and replaced by the global energy).

\section{References}

Chabas, F., Desanti, A. and Soize, C., 1986, "Probabilistic Structural Modeling in Linear Dynamic Analysis of Complex Mechanical Systems. II - Numerical Analysis and Applications", La Recherche Aérospatiale (English edition), Vol. 5, pp. 49-67.

Cuschieri, J.M. and Feit, D., 1995, "Acoustic Scattering from a Fluid-Loaded Elastic Plate with a Distributed Inhomogeneity of Varying Length Scales" J. Acoust. Soc. Am., Vol. 98, No. 5, pp. 2889-2889.

Dyer, I., 1994, "Scattering from Internally Complex Finite Shell Models", J. Acoust. Soc. Am., Vol. 95, No. 5, pp. 2867-2867. 
Feit, D, and Pierce, A.D., 1995, "Vibrations and Acoustical Response of Fuzzy Structures", $J$. Acoust. Soc. Am., Vol. 97, No. 1, pp. 705-705.

Grace, A., 1992, Optimization Toolbox for Use with Matlab, The Math Works Inc., Natick, MA.

Lyon, R. H., 1995, Statistical Energy Analysis and Structural Fuzzy, J. Acoust. Soc. Am., Vol. 97, No. 5, pp. 2878-2881.

Lyon, R. H., and DeJong, R.G., 1995, Theory and Application of Statistical Energy Analysis, Butterworth-Heinemann.

Maidanik, G., 1995, "Power Dissipation in a Sprung Mass Attached to a Master Structure", J. Acoust. Soc. Am., Vol. 98, No. 6, pp. 3527-3533.

Maidanik, G. and Dickey, J., 1995, "A Impulse Response Function for a Fuzzy Structure", J. Acoust. Soc. Am., Vol. 97, No. 3, pp. 1460-1476.

McCoy, J.J., 1994, "The Theory of Fuzzy Structures - A Statistical Continuum Mechanics Interpretation", J. Acoust. Soc. Am., Vol. 95, No. 5, pp. 2846-2846.

Pierce, A. D., 1994, "Mass Per Unit Natural Frequency as a Descriptor of Internal Fuzzy Structure", J. Acoust. Soc. Am., Vol. 95, No. 5, pp. 2845-2845.

Pierce, A.D., 1995a, "Resonant-Frequency-Distribution of Internal Mass Inferred from Mechanical Impedance Matrices", Proceedings of the ASME 15th Biennal Conference on Mechanical Vibration and Noise, ASME, Boston, MA, pp. 229-239.

Pierce, A.D., 1995b, "Fuzzy Elements, their Coupling Rules, and the Jaynes-Shannon Maximum Entropy Principle", J. Acoust. Soc. Am., Vol. 98, No. 5, pp. 2946-2946.

Pierce, A.D., Sparrow, V.W. and Russell, D.A., 1993, "Fundamental Structural-Acoustic Idealizations for Structures with Fuzzy Internals", Paper 93-WA/NCA-17, ASME Winter Annual Meeting, New Orleans, LA, Nov. 28 - Dec. 3. and also in Journal of Vibration and Acoustics, Vol 117, pp. 339-348, 1995.

Photiadis, D.M., 1995, "Scattering from Complex Elastic Structures", J. Acoust. Soc. Am., Vol. 98, No. 5, pp. 2963-2963.

Rochat, J.L. and Sparrow, V.W, 1994, "The Effects of Fuzzy Attachments on Compressional and Shear Waves in a Plate", J. Acoust. Soc. Am., Vol. 95, No. 5, pp. 2847-2847.

Rochat, J.L. and Sparrow, V.W, 1995, "Incorporating Compressional and Shear Wave Types into Fuzzy Structure Models Plates", Proceedings of the ASME 15th Biennal Conference on Mechanical Vibration and Noise, ASME, Boston, MA, pp. 247-252.

Ruckman, C.E., and Feit, D., 1995a, "Fuzzy Structural Analysis: A Simple Example", Proceedings of the 15th International Congress on Acoustics, M. Newman ed., IUPAP, Trondheim, Norway.

Ruckman, C.E., and Feit, D., 1995b, "Tutorial on Soize's Method for Stochastic Modeling in Structural Acoustics (Fuzzy Structure Analysis)", Proceedings of the ASME 15th Biennal Conference on Mechanical Vibration and Noise, ASME, Boston, MA, pp. 241-246.

Russell, D. A., 1995, The Theory of Fuzzy Structures and its Application to Waves in Plates and Shells, The Pennsylvania State University, Graduate Program in Acoustics, State College, Pennsylvania, USA, PhD Thesis.

Russell, D. A. and Sparrow, V.W., 1992, "Acoustic Scattering from a Fluid-Loaded Plate With an Attached Structural Fuzzy", J. Acoust. Soc. Am., Vol. 91, pp. 2440-2440. 
Russell, D. A. and Sparrow, V.W., 1995, "Backscattering from a Baffled Finite Plate Strip with Fuzzy Attachments", J. Acoust. Soc. Am., Vol 98, No 3, 1527-1533.

Soize, C., 1986, "Probabilistic Structural Modeling in Linear Dynamic Analysis of Complex Mechanical Systems. I - Theoretical Elements", La Recherche Aérospatiale (English edition), Vol. 5, pp. 23-48.

Soize, C., 1993, "A Model and Numerical Method in the Medium Frequency Range for Vibroacoustic Predictions Using the Theory of Structural Fuzzy", J. Acoust. Soc. Am., Vol. 94, No. 2, pp. 849-865.

Soize, C., 1995, "Vibration Damping in Low-Frequency Range Due to Structural Complexity. A Model Based on the Theory of Fuzzy Structures and Model Parameters Estimation", Computers and Structures, Vol. 58, No. 5, pp. 901-915.

Soize, C., Desanti, A. and David, J.M., 1992, "Numerical Methods in Elastoacoustics for Low and Medium Frequency Ranges", La Recherche Aérospatiale (English edition), Vol. 5, pp. $25-44$.

Sparrow, V. W., 1991, "Soize's Theory of Structural Fuzzy: An Examination of Fundamental Assumptions", J. Acoust. Soc. Am., Vol 89, pp. 1867-1867.

Sparrow, V. W., 1995, "Time Domain Simulation and Visualization of Fuzzy Structures", $J$. Acoust. Soc. Am., Vol 98, No 5, Pt. 2, pp. 2947-2947.

Sparrow, V. W., Russel, D.A. and Rochat, J.L., 1994, "Implementation of Discrete Fuzzy Structure Models in Mathematica", International Journal for Numerical Methods in Engineering, Vol. 37, pp. 3005-3014.

Steinberg, B.Z. and McCoy, J.J., 1995, "Towards Local Effective Parameter Theories Using Multiresolution Decomposition", J. Acoust. Soc. Am., Vol. 96, No. 2, pp. 1130-1143.

Strasberg, M., 1996, "Continuous Structures as "Fuzzy" Substructures", J. Acoust. Soc. Am., (to appear).

Strasberg, M. and Feit, D., 1996, "Vibration Damping of Large Structures Induced by Attached Small Resonant Structures", J. Acoust. Soc. Am., Vol. 99, No. 1, pp. 335-344. 


\section{LEGENDS ACCOMPANYING EACH FIGURE}

Fig. 1. Geometrical configuration of the master structure coupled with its fuzzy substructures. Fig. 2. Modulus of the acceleration at $x_{1}=7$ for the beam with no oscillators (dashed line) and the beam coupled with the oscillators (irregular solid line).

Fig. 3. Modulus of the acceleration at $x_{1}=15$ for the beam with no oscillators (dashed line) and the beam coupled with the oscillators (irregular solid line).

Fig. 4. Values of $<\Pi_{\text {mast,diss }}^{\mathrm{SEA}}>$ (solid line) and $<\Pi_{\text {mast,in }}^{\mathrm{SEA}}>$ (dashed line) obtained by SEA for the beam coupled with the oscillators.

Fig. 5. Values of $<\Pi_{\text {mast-fuz }}^{\mathrm{SEA}, 1}>$ (dashed line) and $<\Pi_{\text {mast-fuz }}^{\mathrm{SEA}, 2}>$ (solid line) obtained by SEA for the beam coupled with the oscillators.

Fig. 6. Solution of the nonlinear constrained optimization problem: $\left(\nu_{3}^{1}\right)_{\mathrm{opt}, m}$ (dashed line) and $\left(\nu_{3}^{2}\right)_{\text {opt }, m}$ (solid line).

Fig. 7. Modulus (a), real part (b) and imaginary part (c) of the acceleration at $x_{1}=7$ of the beam coupled with the oscillators, corresponding to the reference (irregular solid line) and obtained by the fuzzy structure theory (smoothed solid line).

Fig. 8. Modulus (a), real part (b) and imaginary part (c) of the acceleration at $x_{1}=15$ of the beam coupled with the oscillators, corresponding to the reference (irregular solid line) and obtained by the fuzzy structure theory (smoothed solid line). 


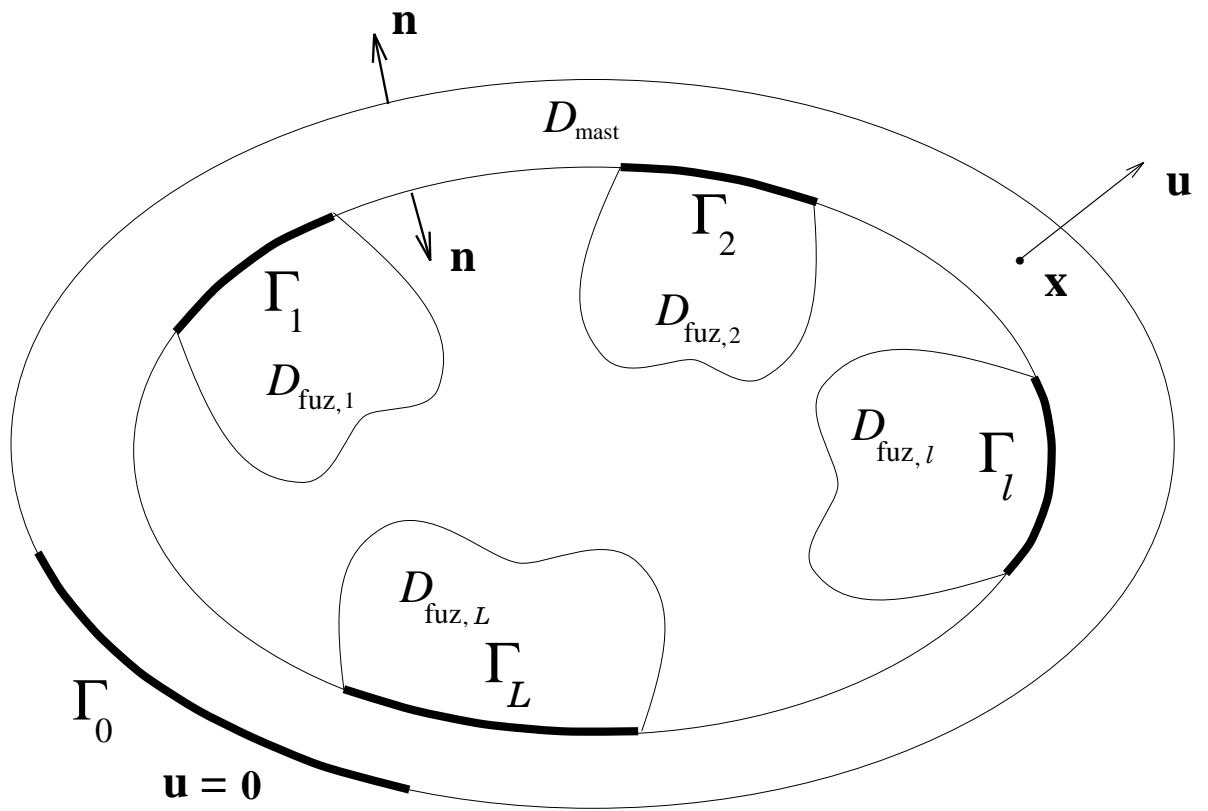

Fig. 1

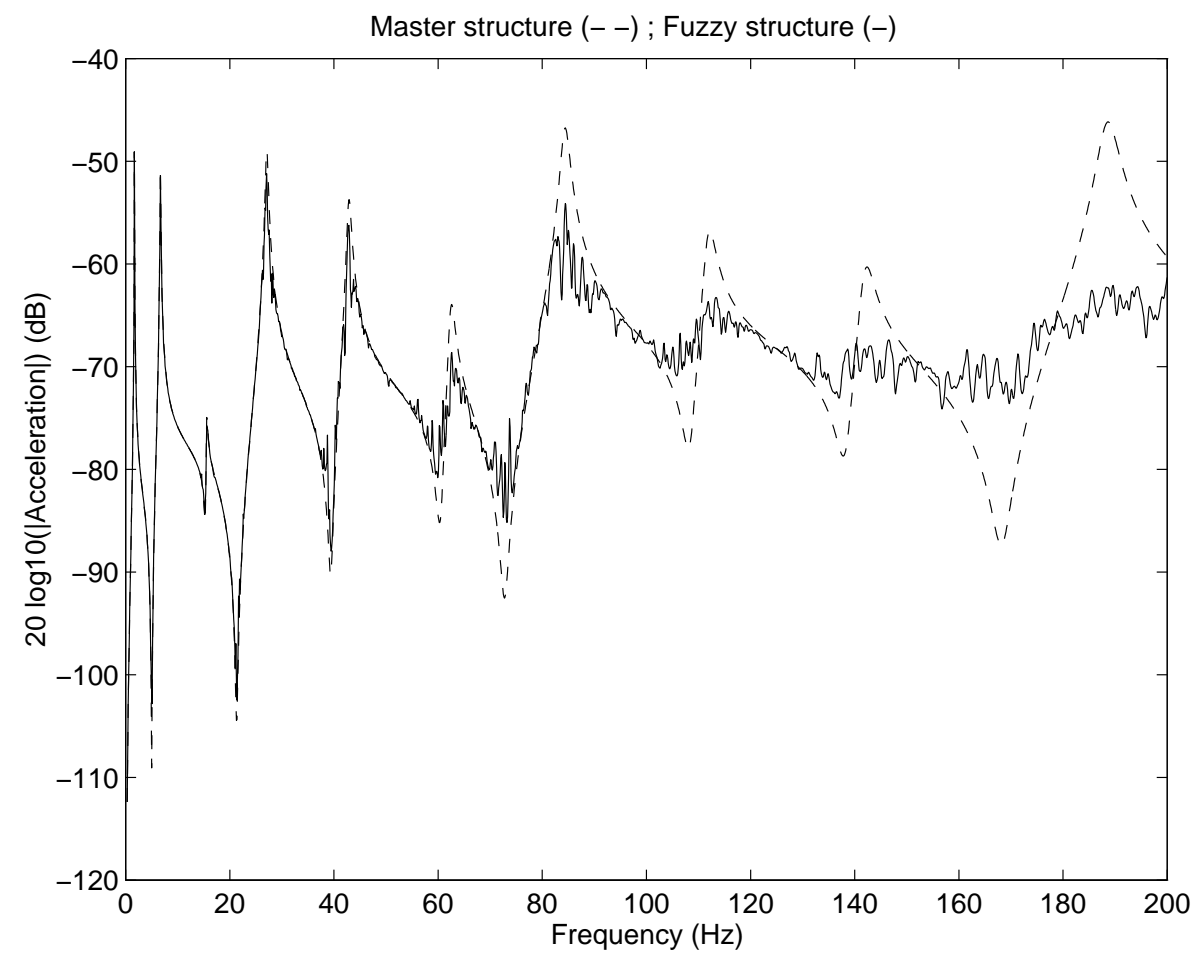

Fig. 2 


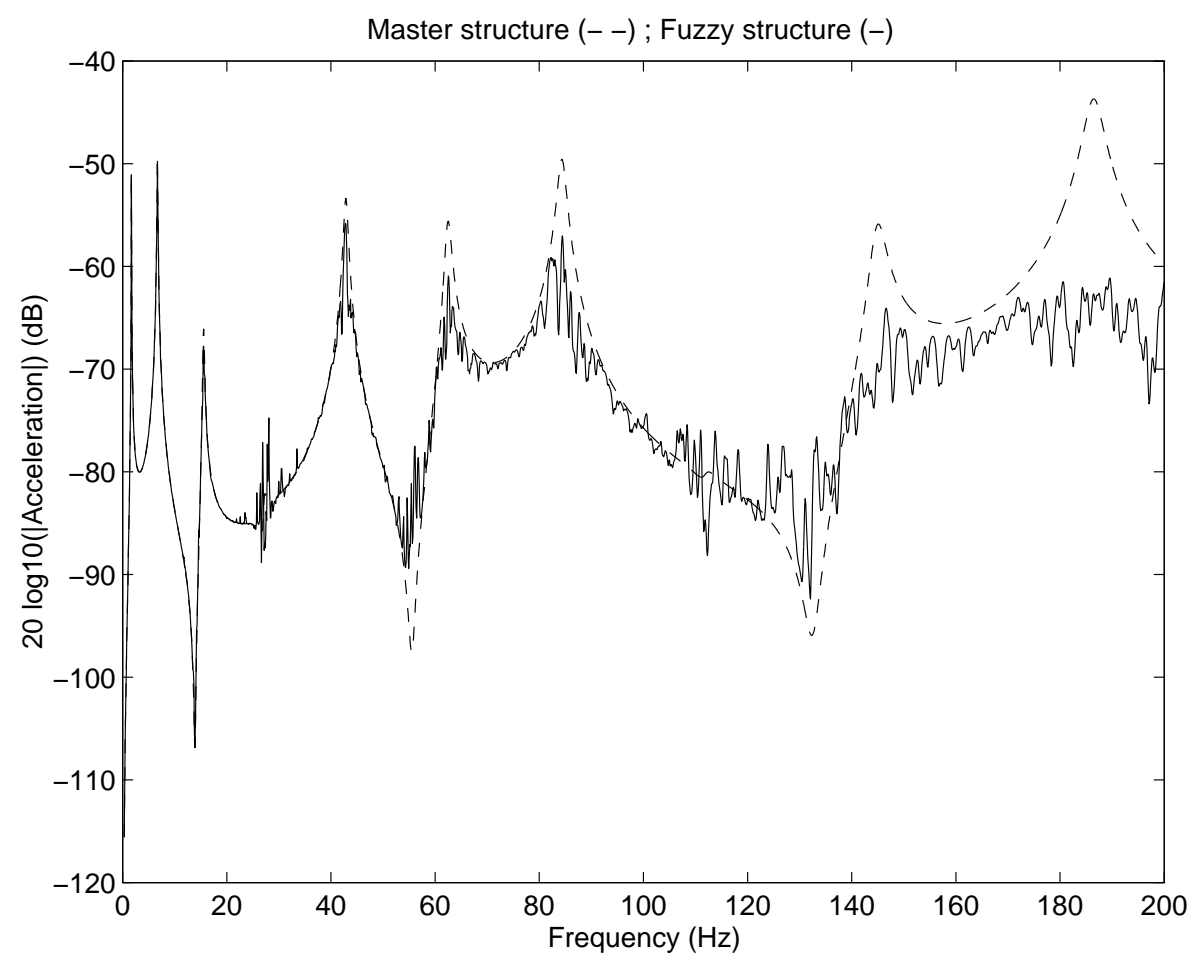

Fig. 3

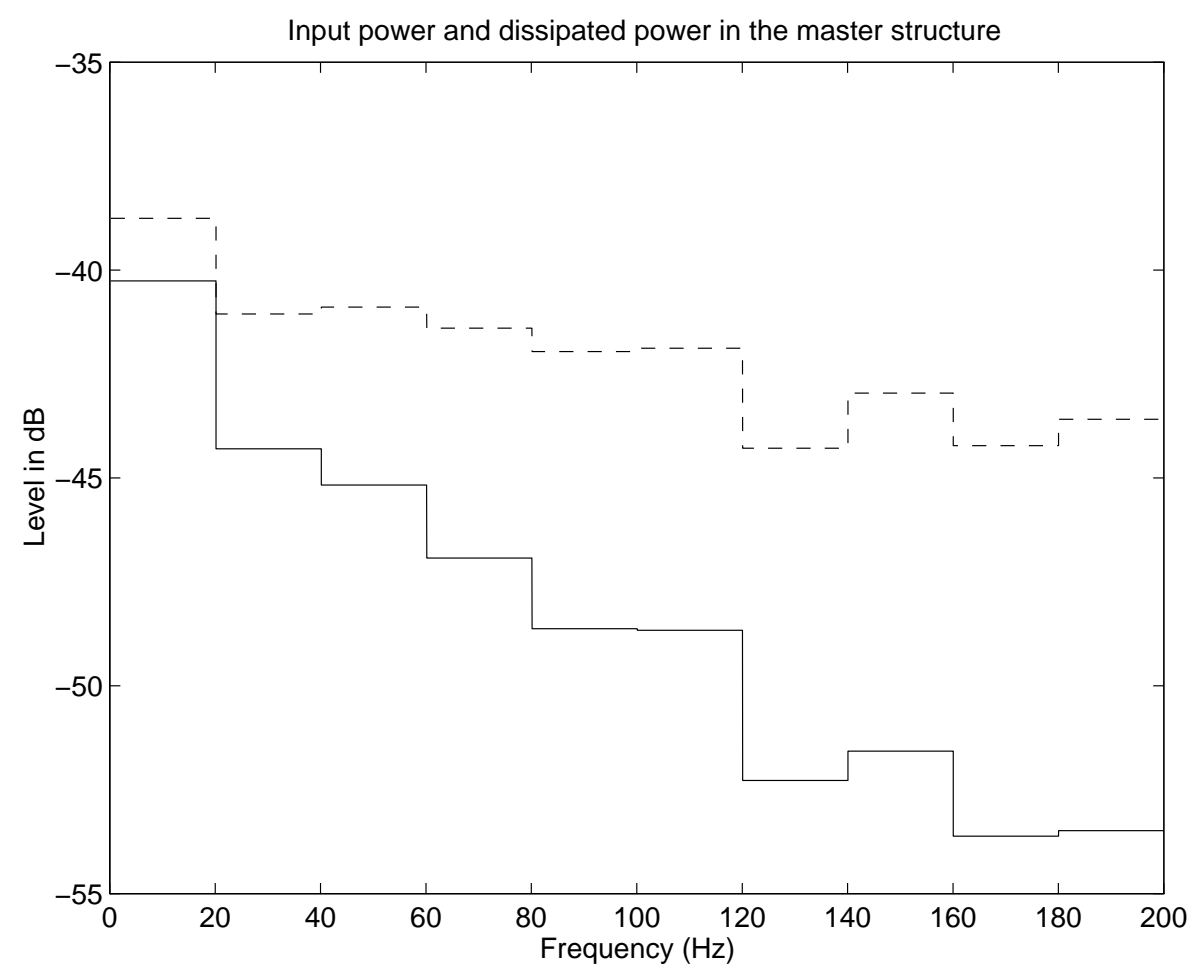

Fig. 4 


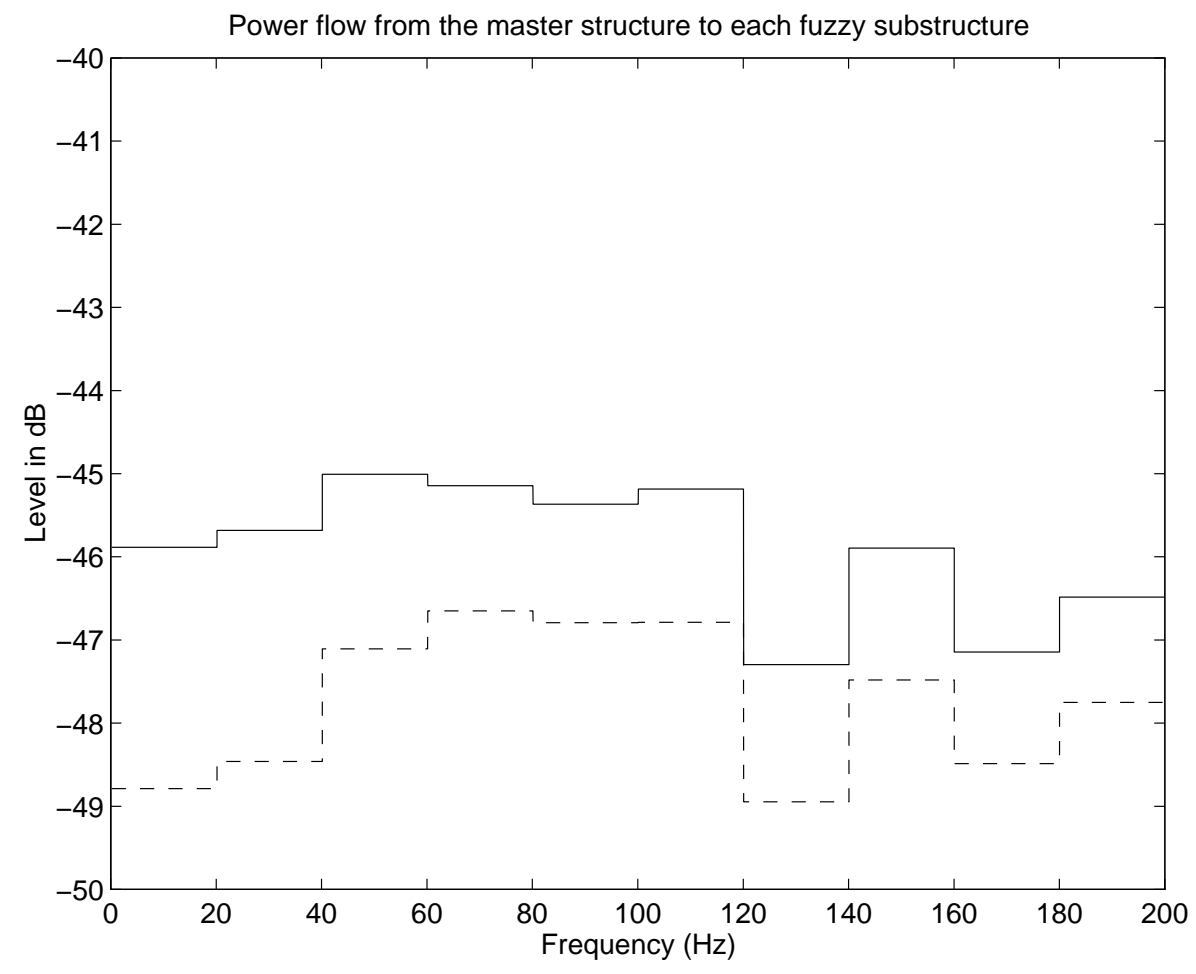

Fig. 5

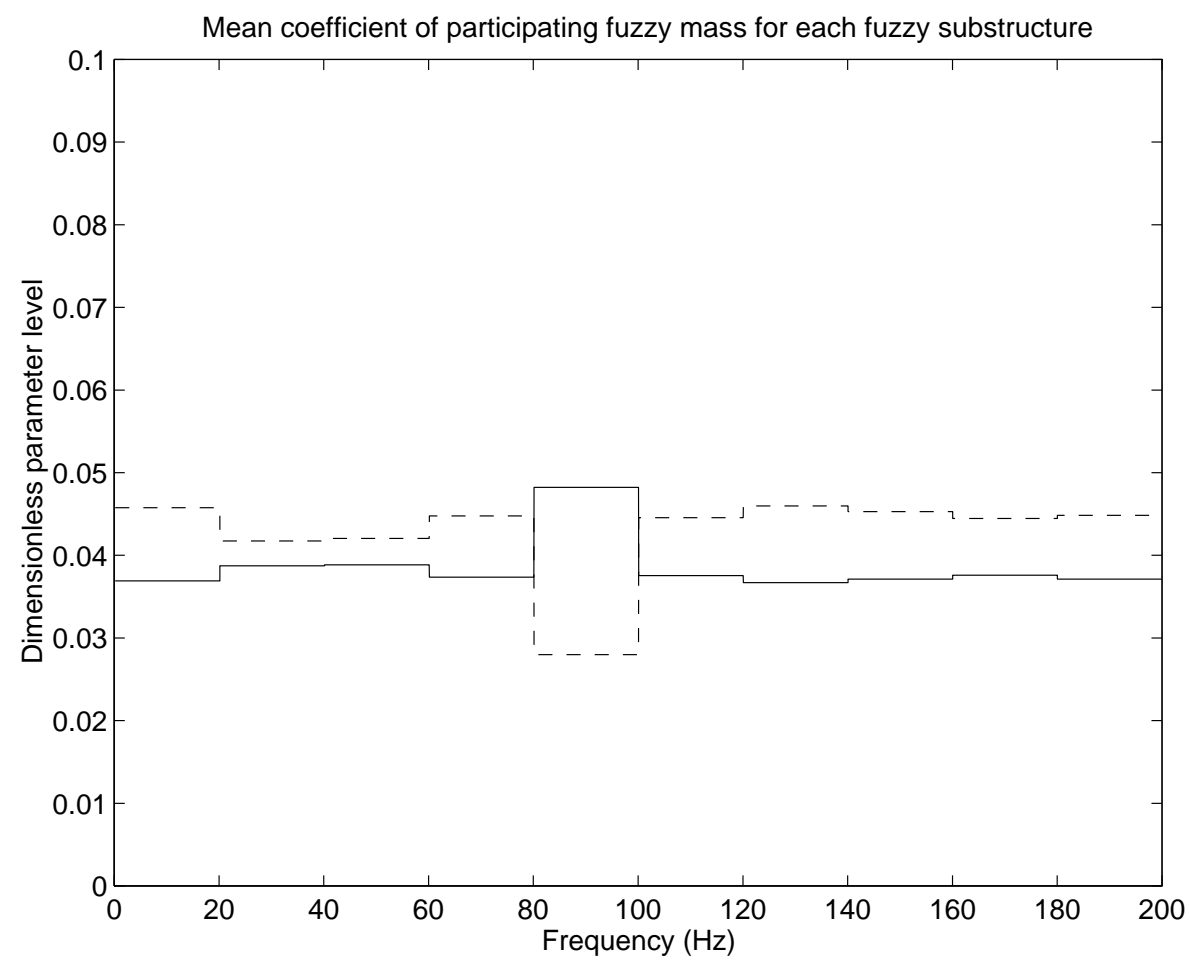

Fig. 6 


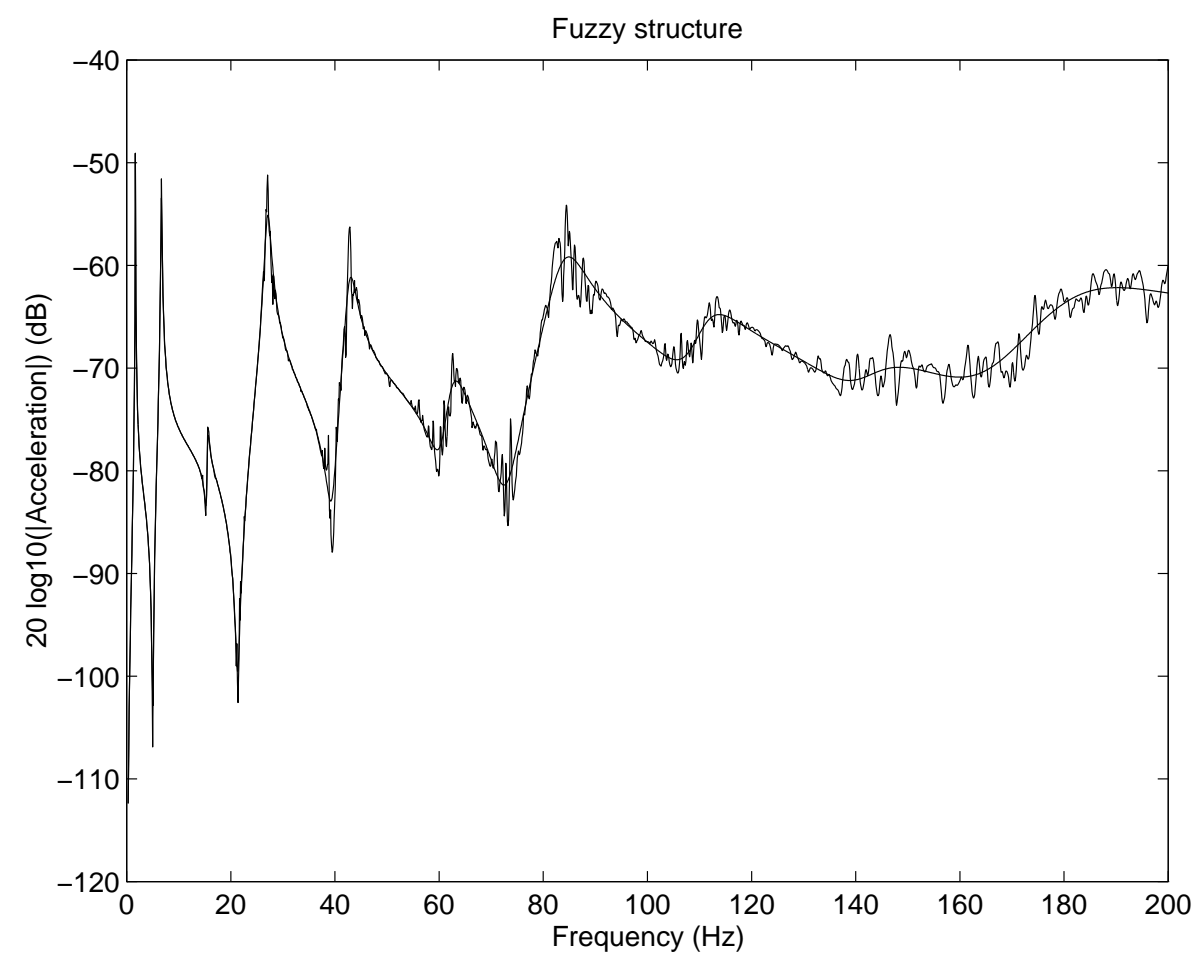

Fig. 7-a

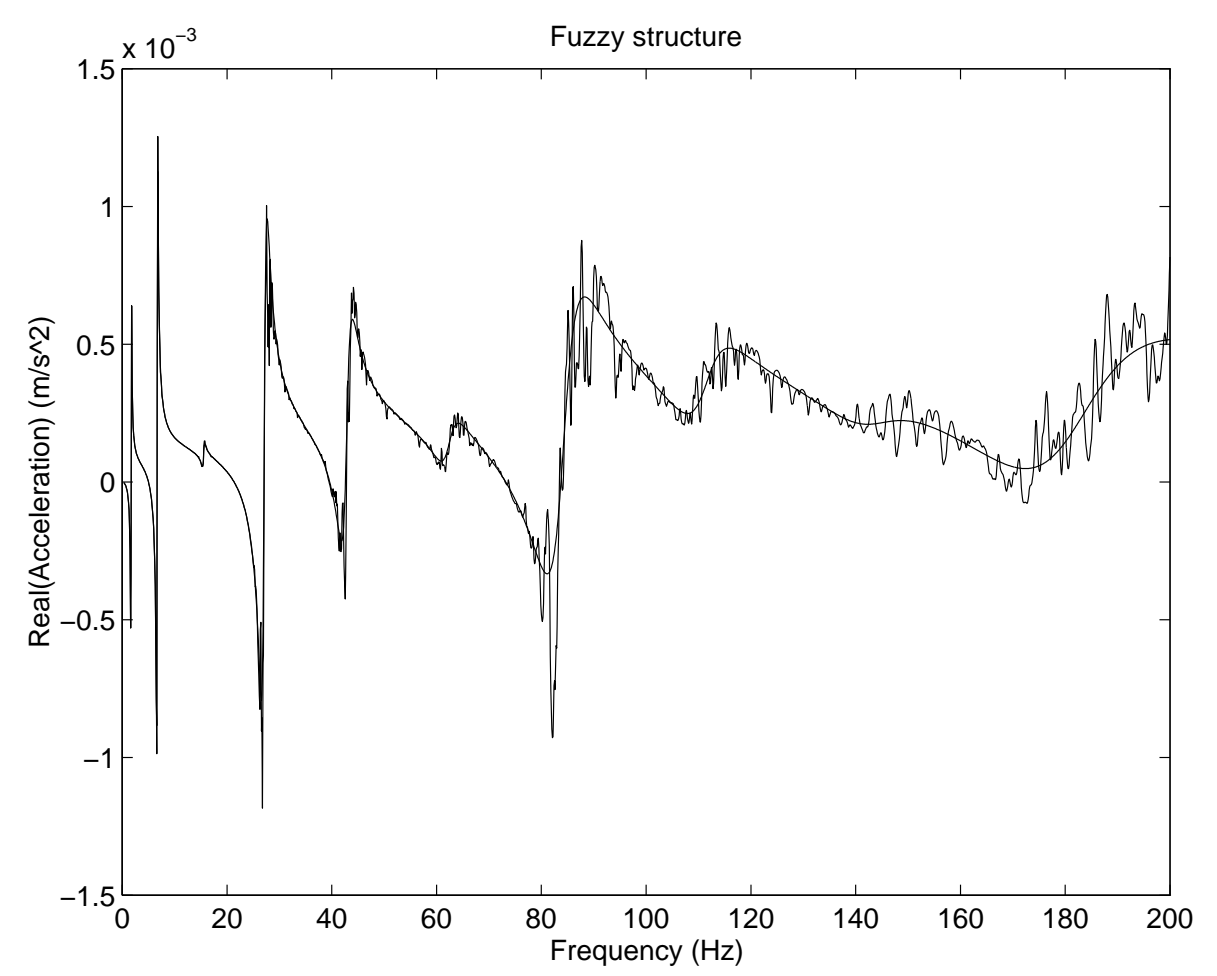

Fig. 7-b 


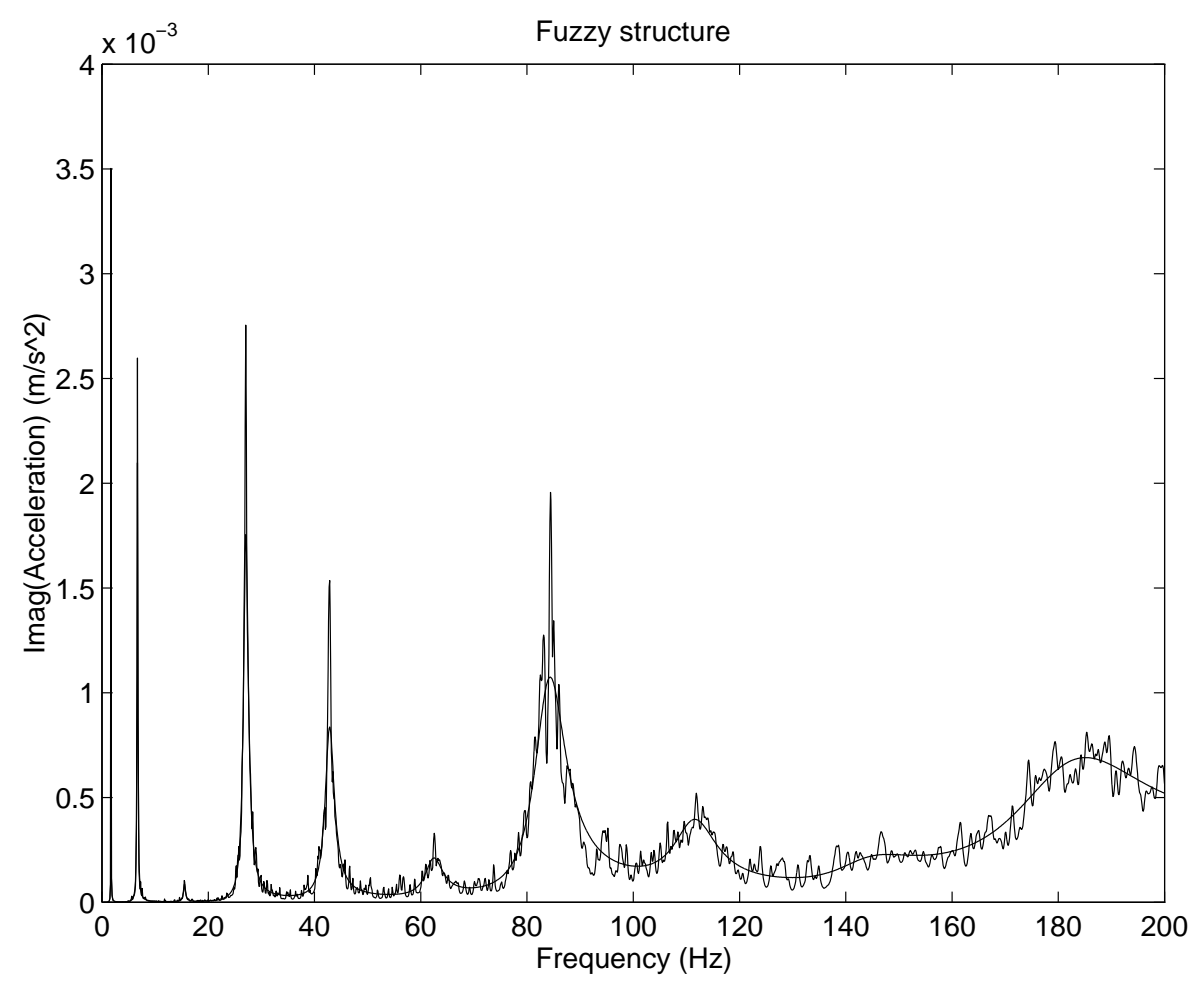

Fig. 7-c

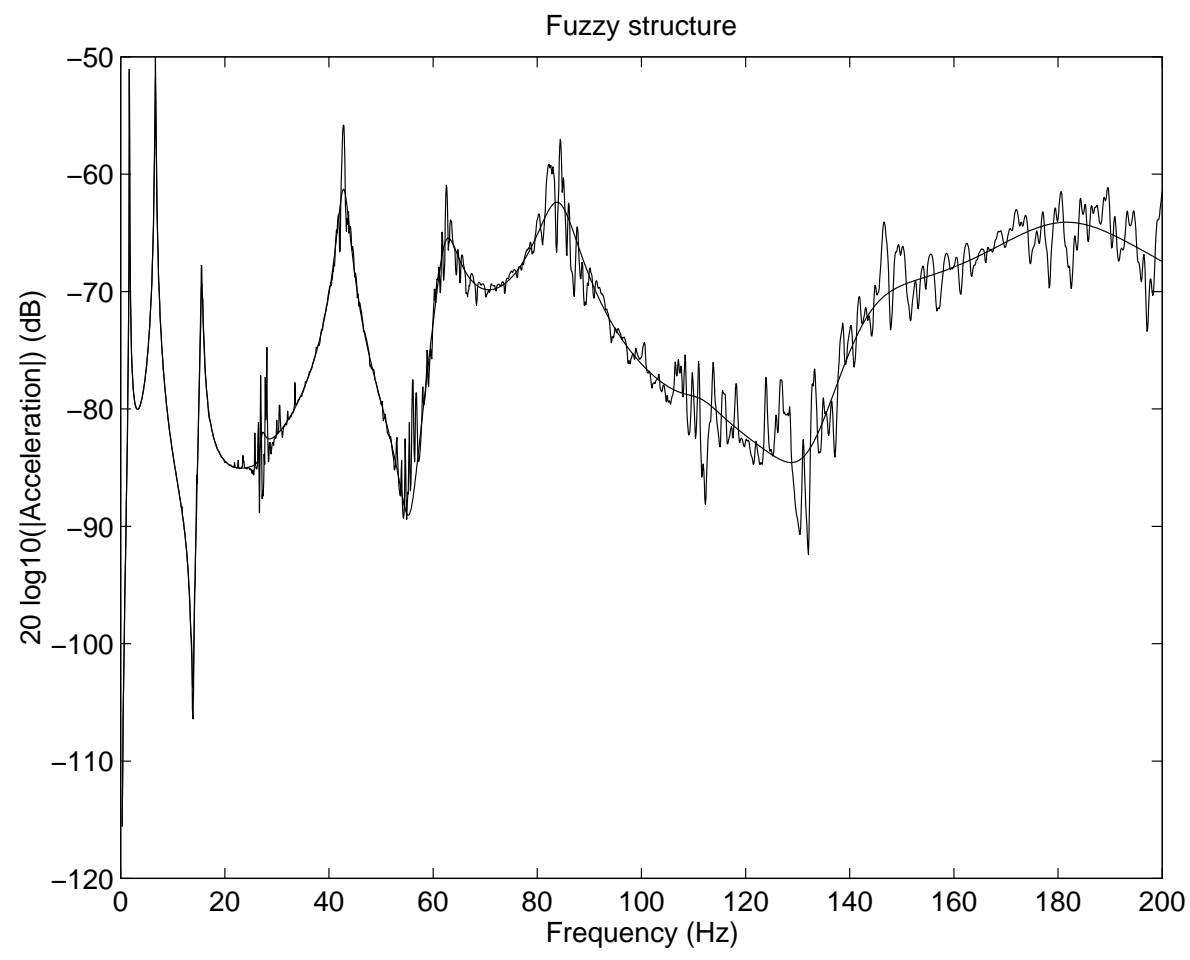

Fig. 8-a 


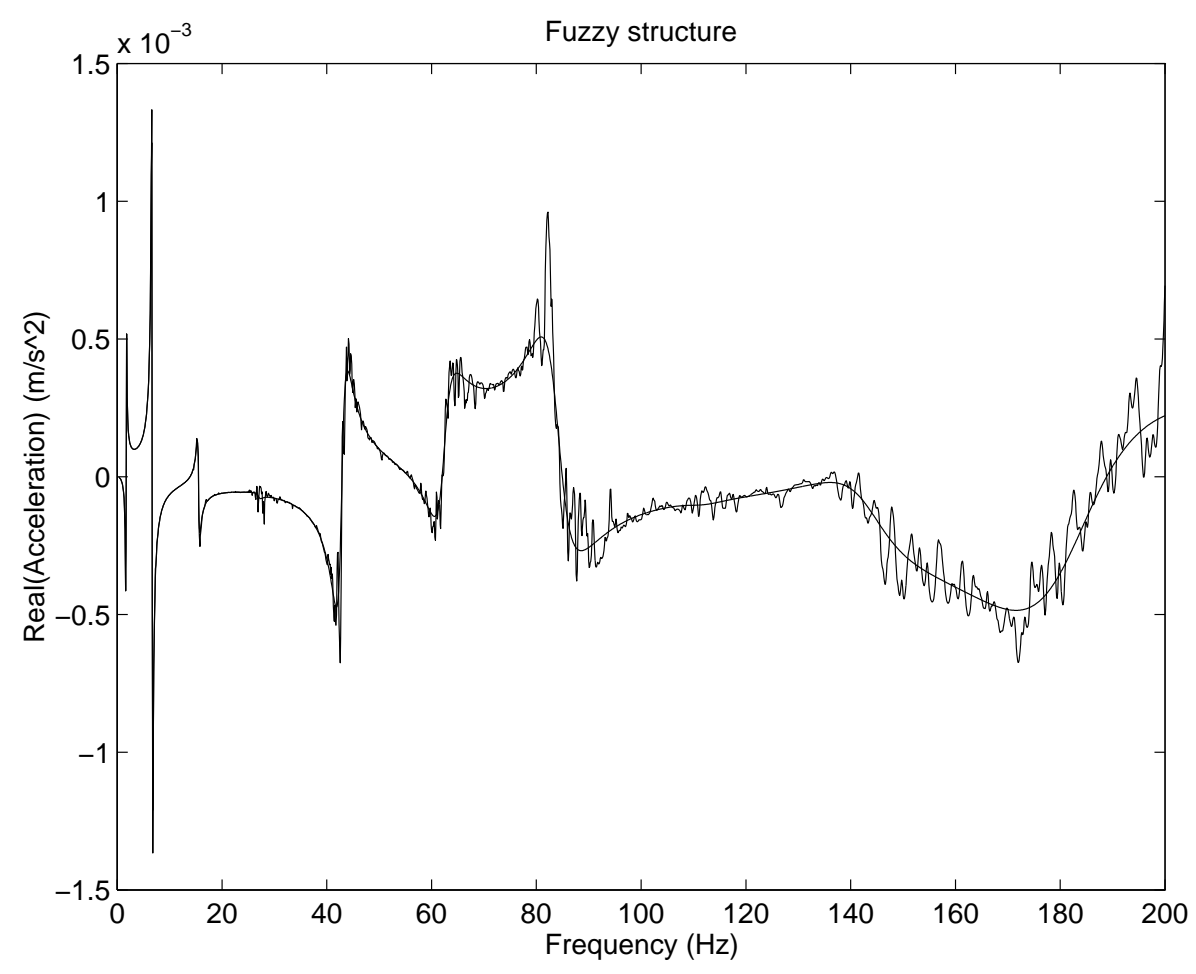

Fig. 8-b

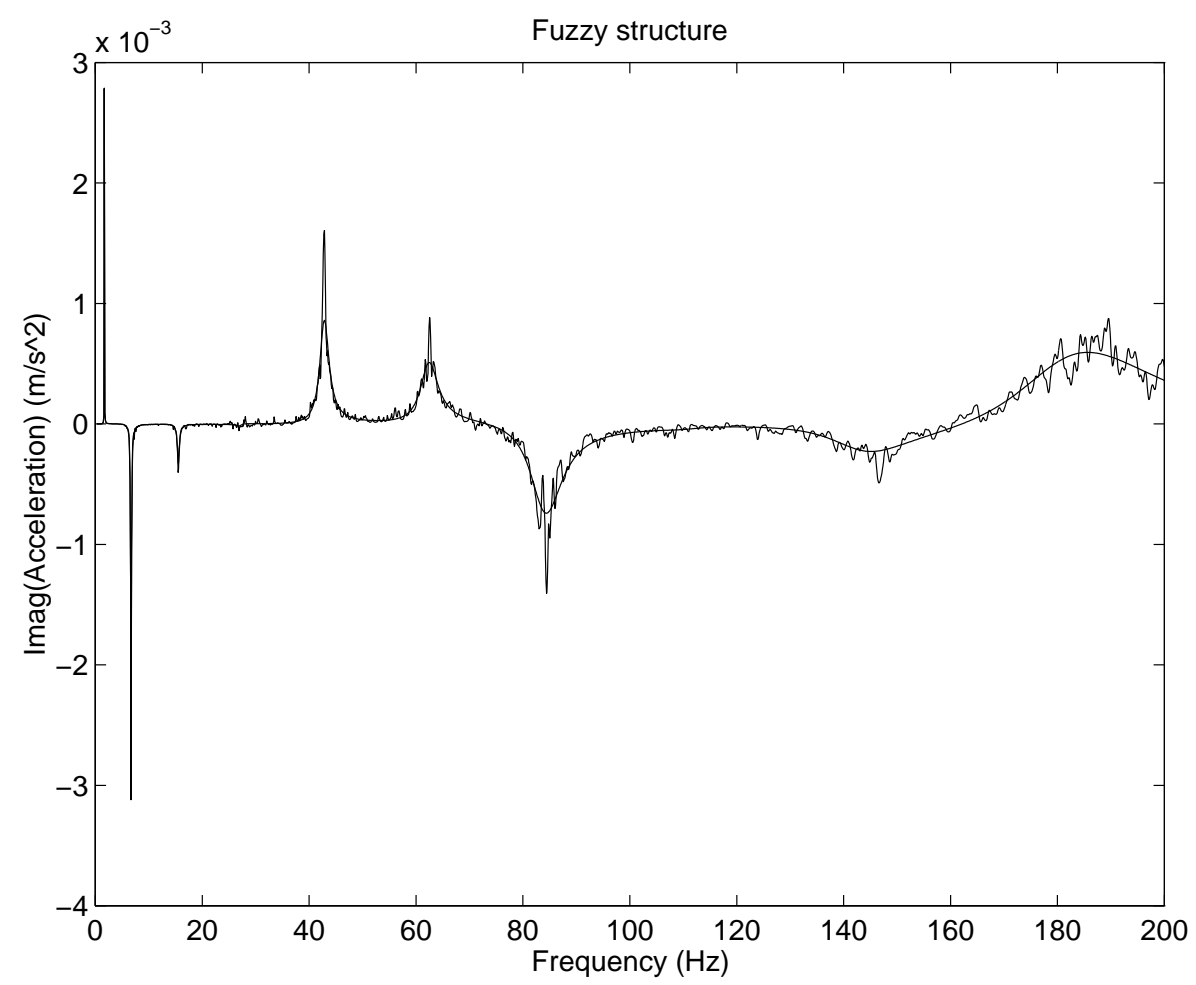

Fig. 8-c 